\title{
MAGNETIZATION SWITCHING ON SMALL FERROMAGNETIC ELLIPSOIDAL SAMPLES
}

\author{
FranÇOis Alouges ${ }^{1}$ And Karine Beauchard ${ }^{2}$
}

\begin{abstract}
The study of small magnetic particles has become a very important topic, in particular for the development of technological devices such as those used for magnetic recording. In this field, switching the magnetization inside the magnetic sample is of particular relevance. We here investigate mathematically this problem by considering the full partial differential model of Landau-Lifschitz equations triggered by a uniform (in space) external magnetic field.
\end{abstract}

Mathematics Subject Classification. 49J15, 35A05, 35A07, 35D05, 35K20, 35K55, 35Q60.

Received December 19, 2007.

Published online July 19, 2008.

\section{INTRODUCTION}

Ferromagnetic materials nowadays are used in numerous technological devices (magnetic recording, cellular phones, etc). Among these applications, magnetic storage is probably one of the most important areas. Devices such as hard-disks, or magnetic RAM are composed of several small ferromagnetic particles capable of being magnetized in two opposite directions, allowing for the storage of one bit of information.

Being able to switch the magnetization in a quick and sure way into this sample is therefore of prime interest. Not surprisingly, the switching of the magnetization in small elongated particles has received a lot of attention (see for instance [2,16] or [3] and references therein) after the pioneering work of Kikuchi [14] where an analytical solution is given in the case of a spherical particle uniformly magnetized.

However, if physicists have worked a lot on such problems by giving strategies to switch the magnetization with the help of an external magnetic field inside nanoscale ferromagnetic particles, the dynamics of the magnetization is usually modelized with a monodomain particle for which the Landau-Lifschitz equation takes the particular form of an ordinary differential equation. This is probably due to the fact that for particles in which the magnetization is not constant, the Landau-Lifschitz equation becomes a non-linear and non local partial differential equation. This equation remains largely badly understood since in all generality, strong solutions are only known to exist locally in time [5] and whenever weak solutions are considered $[1,18]$ although they are defined for all time, such solutions are likely to be nonunique. In this article, we address the question of studying mathematically the possibility of switching the magnetization inside an elongated particle with

\footnotetext{
Keywords and phrases. Landau-Lifschitz equation, micromagnetics, stabilization.

1 Laboratoire de Mathématiques, Bât. 425, Université Paris-Sud XI, 91405 Orsay Cedex, France.

francois.alouges@math.u-psud.fr

2 CMLA, ENS Cachan, CNRS, Universud, 61 Avenue du président Wilson, 94230 Cachan, France.

Karine.Beauchard@cmla.ens-cachan.fr
} 
external magnetic fields that are uniform in space (but may be variable in time). As we shall see, although we will restrict to small ellipsoidal ferromagnetic particles, we will consider the full PDE problem, and both weak and strong solutions.

The magnetization $m$ inside a ferromagnetic body, located in a space domain $\Omega$, is a three dimensional vector field, defined on $\Omega$ and constrained to be of constant magnitude through the sample. After a suitable renormalization, we consider this magnitude to be equal to 1 . The evolution of the magnetization inside a ferromagnetic body is modelized by the Landau-Lifschitz equation,

$$
\frac{\partial m}{\partial t}=\alpha[H(m)-\langle H(m), m\rangle m]-m \wedge H(m), \text { in } \Omega .
$$

Here, $H(m)$ is the total magnetic field induced by several physical phenomena (exchange, stray-field, anisotropy, exterior field), $\alpha>0$ is a damping coefficient which depends on the material (we refer the reader to [4] or [12] for a more complete description of the physical model). In this equation, $\langle.,$.$\rangle denotes the Euclidean scalar$ product on $\mathbb{R}^{3}$ and $\wedge$ is the vectorial product. Equivalently, at least for smooth solutions, the equation (1.1) may be written under the so-called Gilbert form

$$
\frac{\partial m}{\partial t}-\alpha\left(m \wedge \frac{\partial m}{\partial t}\right)=-\left(1+\alpha^{2}\right)(m \wedge H(m))
$$

and under the form,

$$
\alpha \frac{\partial m}{\partial t}+\left(m \wedge \frac{\partial m}{\partial t}\right)=\left(1+\alpha^{2}\right)[H(m)-\langle H(m), m\rangle m] .
$$

For a ferromagnetic body without anisotropy, the magnetic field $H(m)$ can be expressed, in order to emphasize the dependence on the (non-constant in time) external magnetic field, as

$$
H(m)=-\frac{\partial \mathcal{E}}{\partial m}+H_{\mathrm{ext}}
$$

where $\mathcal{E}(m)$ is the micromagnetic energy associated to a given magnetization $m$,

$$
\mathcal{E}(m):=\frac{A}{2} \int_{\Omega}|\nabla m|^{2}-\frac{1}{2} \int_{\Omega}\left\langle H_{d}(m), m\right\rangle
$$

This leads to

$$
H(m):=A \Delta m+H_{d}(m)+H_{\text {ext }},
$$

where $H_{\text {ext }}$ is the uniform in space external magnetic field applied to the sample, $A$ is the so-called exchange constant [4], and $H_{d}(m)$ is the stray field generated by the magnetization $m$ itself via the following dimensionless Maxwell equations

where

$$
\left\{\begin{array}{l}
H_{d}(m)=\nabla \phi, \text { in } \mathbb{R}^{3} \\
\Delta \phi=-\operatorname{div}(\bar{m}), \text { in } \mathbb{R}^{3} \\
H_{d}(m) \text { vanishes at infinity }
\end{array}\right.
$$

$$
\bar{m}=\left\{\begin{array}{l}
m \text { in } \Omega \\
0 \text { in } \mathbb{R}^{3} \backslash \Omega .
\end{array}\right.
$$

It is well known that $H_{d}(m)$ is the $L^{2}\left(\mathbb{R}^{3}\right)$-orthogonal projection of $-\bar{m}$ on gradients from which we deduce

$$
\left\|H_{d}(m)\right\|_{L^{2}\left(\mathbb{R}^{3}\right)} \leqslant\|\bar{m}\|_{L^{2}\left(\mathbb{R}^{3}\right)} .
$$

We will frequently use the following consequence

$$
\forall m \in L^{2}(\Omega),\left\|H_{d}(m)\right\|_{L^{2}(\Omega)} \leqslant\|m\|_{L^{2}(\Omega)} .
$$


The natural boundary conditions are of Neumann type, thus, we will work on the following Cauchy problem

$$
\left\{\begin{array}{l}
\frac{\partial m}{\partial t}=\alpha[H(m)-\langle H(m), m\rangle m]-m \wedge H(m), x \in \Omega, t \in(0, T) \\
\frac{\partial m}{\partial \nu}(t, x)=0, x \in \partial \Omega, t \in(0, T) \\
m(0, x)=m_{0}(x), x \in \Omega
\end{array}\right.
$$

It is a non linear control system in which

- the state is the magnetization $m$, with $m(t): \Omega \rightarrow S^{2}$, for every $t$;

- the control is the external magnetic field $H_{\text {ext }}: t \in \mathbb{R}_{+} \mapsto \mathbb{R}^{3}$.

This means that we seek the possibility of using a time dependent magnetic field $H_{\text {ext }}$ in order to control the magnetization $m$. In this article, we are interested in the existence and the properties of a such control $H_{\text {ext }}$ that steers $m$ from $m(0)=u$ to $m(T)=-u$ where $u$ and $-u$ are global minimizers of the micromagnetic energy $\mathcal{E}$. To further simplify the presentation, we will furthermore assume that the exchange constant $A$ in (1.5) is equal to 1. Let us also quote the paper by Carbou et al. [6] which also treats a control problem in micromagnetics, but in the completely different context of moving a wall in a nano-wire. We will also give a couple of results in the problem similar to (1.9) but posed in 2D. By this, we mean that the domain is bidimensional, but the magnetization still takes values into $S^{2}$. However, the stray field satisfies (1.6) and (1.7) but in $\mathbb{R}^{2}$. This models an infinite ferromagnetic cylindrical rod along the axis of which the solution is invariant. As a consequence of (1.6) and (1.7) the component of the stray field parallel to the axis of the cylinder vanishes.

This article is organized as follows.

In Section 2, we consider a ferromagnetic body having an ellipsoidal shape. Then, the stray field of uniform magnetizations is uniform, thus, a subclass of solutions of (1.9) are uniform magnetizations that solve an ordinary differential equation (ODE) presented in Section 2.1. The goal of Section 2 is the study of the switching for those uniform magnetizations. We may assume that $\pm e_{1}$ are global minimizers of the micromagnetic energy. In Section 2.2, we prove the existence of external magnetic fields $H_{\text {ext }}$ that produce the switching from $m(0)=+e_{1}$ to $m(T)=-e_{1}$ for every $T>0$. Then, we justify the existence of optimal magnetic fields realizing this switching, and we show that the associated solutions $m$ are not 2-dimensional, excepted when $\Omega$ is a sphere. In Section 2.3, we study the cost of the optimal control as $T \rightarrow+\infty$. We prove that this cost converges to zero if and only if there exists two orthogonal global minimizers of the micromagnetic energy.

The Section 3 is dedicated to weak solutions for the partial differential equation (PDE) (1.9). In Section 3.1, we prove the existence of weak solutions of (1.9). In Section 3.2, we study their convergence to uniform magnetizations when the size of the domain $\Omega$ goes to zero. This already shows that the external field found in Section 2 allows an approximate switching on any sufficiently small domain in the very general sense of weak solutions. To go further, we need more regularity and strong solutions. This imposes restrictions on either the shape of the domain or the regularity and smallness of the initial condition. Namely, Section 4 is dedicated to smooth solutions of (1.9). In Section 4.1, we present preliminary results useful for the proof done in the next section. In Section 4.2, we prove the existence and uniqueness of local (in time) smooth solutions when $\Omega$ is a bounded domain of $\mathbb{R}^{2}$ or $\mathbb{R}^{3}$. Then, in Section 4.3, we prove that such local solutions indeed provide global solutions when $\Omega$ is a $2 \mathrm{D}$ bounded domain and when the initial condition is in a $H^{1}$-neighborhood of constant magnetizations. In Section 4.4, we prove the existence of global smooth solutions when $\Omega$ is a small $3 \mathrm{D}$ ellipsoid domain and when the initial condition is in a $H^{2}$-neighborhood of constant magnetizations. Contrarily to the preceding results where we follow the strategy developed by [5], the latter result involves ideas completely different.

In Section 5, we work with small 2D or $3 \mathrm{D}$ ellipsoid domains $\Omega$. We propose explicit external fields that exponentially stabilize the uniform stationary solutions.

In Section 6, we propose a way to realize the approximate switching of PDE solutions on small 2D or 3D ellipsoidal domains. 
In all this article we will use the following notations. The family $\left(e_{1}, e_{2}, e_{3}\right)$ is the canonical basis of $\mathbb{R}^{3}$, if $x \in \mathbb{R}^{3}$, we write its components $x^{(1)}, x^{(2)}$ and $x^{(3)}$. The same letter $C$ denotes different constants that can change from one line to another. Whenever possible, we have explicited the parameters on which those constants depend. When $\Omega$ is an open bounded subset of $\mathbb{R}^{2}$ or $\mathbb{R}^{3}$ and $T>0, Q_{T}$ denotes $(0, T) \times \Omega$. Eventually, for every map $f: \Omega \rightarrow \mathbb{R}^{3}$, we denote by $f_{\sharp}$ its space average

$$
f_{\sharp}:=\frac{1}{|\Omega|} \int_{\Omega} f(x) \mathrm{d} x .
$$

\section{Magnetization SWitching on ellipsoidal domains: ODE study}

\subsection{A simplified Landau-Lifschitz equation}

It is well known that, when $\Omega$ is a $3 \mathrm{D}$ ellipsoidal domain over which the magnetization is constant, the stray field is also constant on $\Omega$ and therefore satisfies

$$
H_{d}(m)=-D m \text { on } \Omega
$$

where $D$ is a symmetric positive matrix. Up to an orthonormal change of coordinates, we may take

$$
D:=\left(\begin{array}{ccc}
\alpha_{1} & 0 & 0 \\
0 & \alpha_{2} & 0 \\
0 & 0 & \alpha_{3}
\end{array}\right),
$$

where $0 \leqslant \alpha_{1} \leqslant \alpha_{2} \leqslant \alpha_{3} \leqslant 1$ depend on the size of the three axis of the ellipsoid (the last inequality comes from (1.8)).

In this case, the Landau-Lifschitz equations becomes the ordinary differential system

$$
\left\{\begin{array}{l}
\frac{\mathrm{d} m}{\mathrm{~d} t}=\alpha\left[H_{0}(m)-\left\langle H_{0}(m), m\right\rangle m\right]-m \wedge H_{0}(m), \\
m(0)=m_{0} \\
m: \mathbb{R}_{+} \rightarrow S^{2}
\end{array}\right.
$$

where

$$
H_{0}(m)=-D m+H_{\text {ext }} .
$$

The existence of solutions is a classical matter.

Proposition 2.1. For every $H_{\mathrm{ext}} \in L_{\mathrm{loc}}^{1}\left(\mathbb{R}_{+}, \mathbb{R}^{3}\right)$, for every $m_{0} \in S^{2}$, there exist $T>0$ and a unique function $m \in C^{0}\left([0, T), \mathbb{R}^{3}\right)$, such that,

$$
m(t)=m_{0}+\int_{0}^{t}\left\{\alpha\left[H_{0}(m)-\left\langle H_{0}(m), m\right\rangle m\right]-m \wedge H_{0}(m)\right\} \mathrm{d} \tau,
$$

for every $t \in[0, T)$.

Moreover, if $H_{\mathrm{ext}} \in L_{\mathrm{loc}}^{2}\left(\mathbb{R}_{+}, \mathbb{R}^{3}\right)$, then, $m \in H^{1}\left((0, T), \mathbb{R}^{3}\right)$, the first equation of $(2.1)$ holds in $L^{2}\left((0, T), \mathbb{R}^{3}\right)$ and $|m| \equiv 1$.

If $H_{\mathrm{ext}} \in C^{0}\left(\mathbb{R}_{+}, \mathbb{R}^{3}\right)$, then $m \in C^{1}\left([0,+\infty), S^{2}\right)$, and the first equation of $(2.1)$ holds for every $t \in[0,+\infty)$.

\subsection{Optimal control}

Viewing $H_{\text {ext }}$ as a control parameter, (2.1) turns out to be a flat system i.e. for every reference path $m_{\text {ref }} \in H^{1}\left((0, T), S^{2}\right)$ (for some $\left.T>0\right)$, there exists an external field $H_{\text {ext }}\left[m_{\text {ref }}(t)\right] \in L^{2}\left((0, T), \mathbb{R}^{3}\right)$ such that 
the unique solution $m$ of (2.1) with initial condition $m_{0}=m_{\text {ref }}(0)$, and external field $H_{\text {ext }}\left(m_{\text {ref }}\right)$ coincides with $m_{\text {ref }}$

Indeed, (2.1) rewrites as

$$
m(t)=m_{\mathrm{ref}}(t), \quad \forall t \in(0, T) .
$$

$$
\alpha \frac{\mathrm{d} m}{\mathrm{~d} t}+m \wedge \frac{\mathrm{d} m}{\mathrm{~d} t}=\left(1+\alpha^{2}\right)\left[-D m+H_{\mathrm{ext}}+\left\langle D m-H_{\text {ext }}, m\right\rangle m\right],
$$

thus, for every path $m \in H^{1}\left((0, T), S^{2}\right)$, the magnetic field

$$
H_{\text {ext }}(m):=\frac{1}{1+\alpha^{2}}\left\{\alpha \frac{\mathrm{d} m}{\mathrm{~d} t}+m \wedge \frac{\mathrm{d} m}{\mathrm{~d} t}\right\}+D m-\langle D m, m\rangle m
$$

belongs to $L^{2}\left((0, T), \mathbb{R}^{3}\right)$ and, since $\left\langle H_{\text {ext }}(m), m\right\rangle=0$, allows to follow $m$. The other solutions are such that $\tilde{H}_{\text {ext }}-\left\langle\tilde{H}_{\text {ext }}, m\right\rangle m=H_{\text {ext }}(m)$, and therefore $H_{\text {ext }}(m)$ has the minimal $L^{2}\left((0, T), \mathbb{R}^{3}\right)$-norm, among all possible solutions.

For $T>0$, we introduce the set

$$
V_{T}:=\left\{m \in H^{1}\left((0, T), S^{2}\right) ; m(0)=e_{1}, m(T)=-e_{1}\right\}
$$

and the functional $J_{T}: V_{T} \rightarrow \mathbb{R}_{+}$,

$$
J_{T}(m):=\int_{0}^{T}\left|H_{\text {ext }}(m)(t)\right|^{2} \mathrm{~d} t .
$$

Proposition 2.2. Let $T>0$. There exists a solution $m^{\mathrm{opt}, T} \in V_{T}$ of the minimization problem

$$
J_{T}\left(m^{\mathrm{opt}, T}\right)=\min \left\{J_{T}(\xi), \xi \in V_{T}\right\} .
$$

This solution is not unique and satisfies

$$
\left\{\begin{array}{l}
\frac{2}{1+\alpha^{2}}\left\{-\frac{\mathrm{d}^{2} m}{\mathrm{~d} t^{2}}+\frac{\mathrm{d}}{\mathrm{d} t}[m \wedge D m]+\frac{\mathrm{d} m}{\mathrm{~d} t} \wedge D m+D\left(m \wedge \frac{\mathrm{d} m}{\mathrm{~d} t}\right)\right\} \\
m(0)=e_{1}, m(T)=-e_{1},
\end{array}\right.
$$

where $\lambda:[0, T] \rightarrow \mathbb{R}$.

If $\alpha_{1}=\alpha_{2}=\alpha_{3}$, then any 2 dimensional path

$$
m_{\theta}(t):=\cos \left(\frac{\pi t}{T}\right) e_{1}+\sin \left(\frac{\pi t}{T}\right)\left[\cos (\theta) e_{2}+\sin (\theta) e_{3}\right], \quad \theta \in(0,2 \pi),
$$

is optimal, otherwise, no optimal solution can be only 2 dimensional.

Proof of Proposition 2.2. Let $T>0$. We have, for every $m \in V_{T}$,

$$
J_{T}(m)=\int_{0}^{T} \frac{1}{1+\alpha^{2}}\left|\frac{\mathrm{d} m}{\mathrm{~d} t}\right|^{2}+\frac{2}{1+\alpha^{2}}\left\langle m \wedge \frac{\mathrm{d} m}{\mathrm{~d} t}, D m\right\rangle+|D m-\langle D m, m\rangle m|^{2} .
$$

The existence of an optimal path is a consequence of the direct method of calculus of variations.

For $m=\left(m^{(1)}, m^{(2)}, m^{(3)}\right)^{t} \in V_{T}$, we define

$$
\widetilde{m}=\left(m^{(1)},-m^{(2)},-m^{(3)}\right)^{t} .
$$


An easy calculation gives $J_{T}(m)=J_{T}(\widetilde{m})$, showing that the solution to (2.6) cannot be unique.

The equation (2.7) is the Euler-Lagrange equation of the optimization problem (2.6).

Moreover, any function $m \in V_{T}$ solution of (2.7) satisfies at time 0

$$
\frac{2}{1+\alpha^{2}} \frac{\mathrm{d}^{2} m}{\mathrm{~d} t^{2}}(0)=\frac{2}{1+\alpha^{2}}\left(2 \alpha_{1}-\alpha_{2}-\alpha_{3}\right) \frac{\mathrm{d} m}{\mathrm{~d} t}(0) \wedge e_{1}-\left(\lambda+2 \alpha_{1}^{2}\right) e_{1}
$$

Let $m$ be an optimal path from $e_{1}$ to $-e_{1}$ on $[0, T]$. It is clear from $(2.7)$ that $m \in \mathcal{C}^{1}\left([0, T], S^{2}\right)$ and we have

$$
\forall t \in[0, T], \frac{\mathrm{d} m}{\mathrm{~d} t}(t) \perp m(t) .
$$

Now, since $m$ is not constant, the Cauchy-Lipschitz theorem ensures that

$$
\frac{\mathrm{d} m}{\mathrm{~d} t}(0) \neq 0
$$

and this vector is orthogonal to $m(0)=e_{1}$ because $|m| \equiv 1$. Hence,

$$
\frac{\mathrm{d}^{2} m}{\mathrm{~d} t^{2}}(0) \wedge e_{1}=-\left(2 \alpha_{1}-\alpha_{2}-\alpha_{3}\right) \frac{\mathrm{d} m}{\mathrm{~d} t}(0)
$$

Therefore, in the case $2 \alpha_{1}-\alpha_{2}-\alpha_{3} \neq 0$, the optimal path can not be 2-dimensional.

Now, let us assume that $\alpha_{1}=\alpha_{2}=\alpha_{3}$. The Euler equation (2.7) reduces to

$$
-\frac{2}{1+\alpha^{2}} \frac{\mathrm{d}^{2} m}{\mathrm{~d} t^{2}}=\left(\lambda+2 \alpha_{1}^{2}\right) m
$$

which is the equation of geodesics on the sphere. Those geodesics are bi-dimensional.

Remark 2.1. When $\alpha_{1}<\alpha_{2}=\alpha_{3}$, for every $m=\left(m^{(1)}, m^{(2)}, m^{(3)}\right)^{t} \in H^{1}\left((0, T), \mathbb{R}^{3}\right)$, the function $\tilde{m}:=$ $\left(m^{(1)},-m^{(3)}, m^{(2)}\right)$ satisfies $J_{T}(m)=J_{T}(\tilde{m})$. Thus, the optimal path is not unique, even up to the symmetry defined by (2.10).

However, when $\alpha_{1}<\alpha_{2}<\alpha_{3}$, the uniqueness of the optimal path, up to the symmetry defined in (2.10), is an open problem.

As a sake of example, we provide hereafter the solution obtained by a numerical method based on a shooting strategy, when one solves the boundary value problem $(2.7)$ with $D=\operatorname{diag}(0.02,0.5,1)$. The three components of the magnetization are shown in Figure 1 where it is clear that the solution is not bidimensional only.

Remark 2.2. Of course, minimizing $J_{T}$ over $V_{T}$ leads to discontinuous external fields, which, in practice might be undesirable. Other functionals can be chosen ensuring more regular $H_{\text {ext }}$, for instance, minimizing

$$
\widetilde{J}_{T}(m):=\int_{0}^{T}\left|\frac{\mathrm{d}}{\mathrm{d} t} H_{\text {ext }}(m)\right|^{2} \mathrm{~d} t
$$

over

$$
\widetilde{V}_{T}:=\left\{m \in H^{2}\left((0, T), S^{2}\right) ; m(0)=e_{1}, m(T)=-e_{1}, \frac{\mathrm{d} m}{\mathrm{~d} t}(0)=\frac{\mathrm{d} m}{\mathrm{~d} t}(T)=0\right\}
$$

leads to optimal external fields in $H_{0}^{1}\left((0, T), \mathbb{R}^{3}\right)\left(H_{\text {ext }}(0)=H_{\text {ext }}(T)=0\right.$ corresponds to a switch off for the magnetic source). 

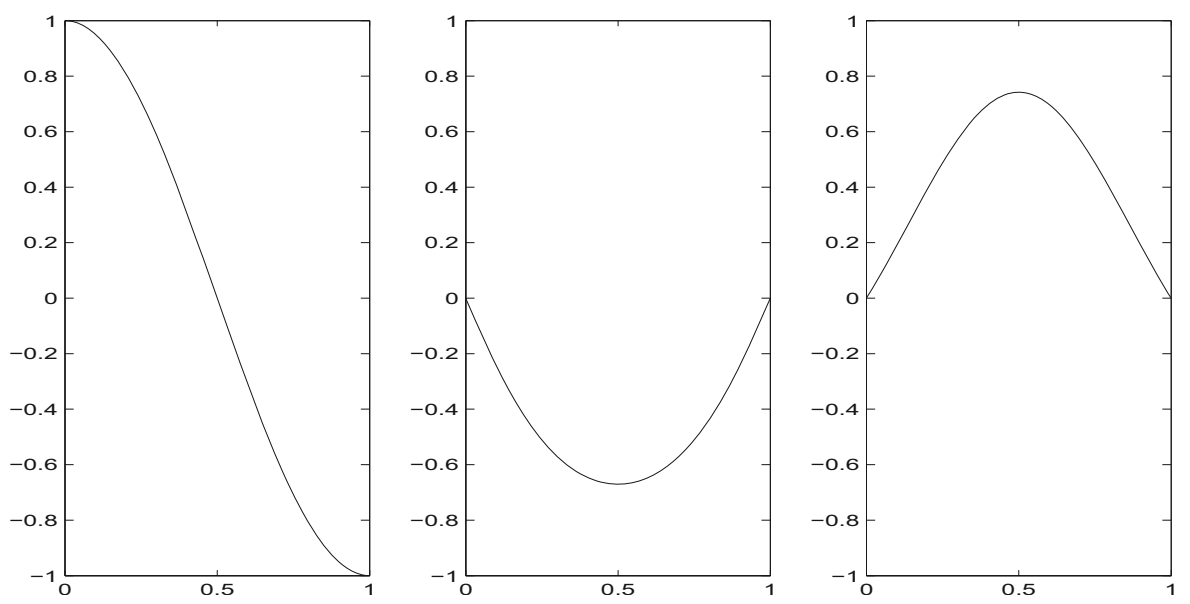

FiguRE 1. The three components of the optimal magnetization with respect to time.

\subsection{Behavior of the optimal cost as $\boldsymbol{T} \rightarrow+\infty$}

The asymptotic behavior, when $T \rightarrow+\infty$, of the optimal cost

$$
f(T):=J_{T}\left(m^{\mathrm{opt}, T}\right)
$$

is given by the following proposition.

Proposition 2.3. If $\alpha_{1}=\alpha_{2} \leqslant \alpha_{3}$, then $f(T) \rightarrow 0$, when $T \rightarrow+\infty$. Otherwise,

$$
f(T) \geqslant 4\left(\frac{1}{1+\alpha^{2}}-\frac{1}{\left(1+\alpha^{2}\right)^{3 / 2}}\right)\left(\alpha_{2}-\alpha_{1}\right),
$$

thus $f(T)$ does not converge to zero when $T \rightarrow+\infty$.

Proof of Proposition 2.3. When $\alpha_{1}=\alpha_{2} \leqslant \alpha_{3}$, computing explicitly the energy of the path (2.8), for $\theta=0$, we get

$$
f(T) \leqslant J_{T}\left(m_{0}\right)=\frac{1}{1+\alpha^{2}} \frac{\pi^{2}}{T}
$$

proving that $f(T) \rightarrow 0$ when $T \rightarrow+\infty$.

Now, let us assume that $\alpha_{1}<\alpha_{2} \leqslant \alpha_{3}$. Let $T>0$ and $m \in V_{T}$. We have

$$
\begin{aligned}
\int_{0}^{T} \frac{2}{1+\alpha^{2}}\left\langle m \wedge \frac{\mathrm{d} m}{\mathrm{~d} t}, D m\right\rangle & =\int_{0}^{T} \frac{2}{1+\alpha^{2}}\left\langle m \wedge \frac{\mathrm{d} m}{\mathrm{~d} t}, D m-\langle D m, m\rangle m\right\rangle \\
& \leqslant \int_{0}^{T} \frac{1}{\left(1+\alpha^{2}\right)^{3 / 2}}\left|\frac{\mathrm{d} m}{\mathrm{~d} t}\right|^{2}+\frac{1}{\left(1+\alpha^{2}\right)^{1 / 2}}|D m \wedge m|^{2}
\end{aligned}
$$

We introduce the notations

$$
\begin{gathered}
C(\alpha):=\frac{1}{1+\alpha^{2}}-\frac{1}{\left(1+\alpha^{2}\right)^{3 / 2}}, \\
T_{1}:=\sup \left\{t>0 ; m^{(1)}(\tau)>0, \quad \forall \tau \in(0, t)\right\},
\end{gathered}
$$


then $m\left(T_{1}\right) \in \operatorname{Span}\left(e_{2}, e_{3}\right)$. We deduce from (2.9) and the last inequality that

$$
\begin{aligned}
J_{T}(m) & \geqslant C(\alpha) \int_{0}^{T}\left|\frac{\mathrm{d} m}{\mathrm{~d} t}\right|^{2}+|D m \wedge m|^{2} \\
& \geqslant 2 C(\alpha) \int_{0}^{T}\left|\left\langle\frac{\mathrm{d} m}{\mathrm{~d} t}, D m-\langle D m, m\rangle m\right\rangle\right| \\
& =2 C(\alpha)\left(\int_{0}^{T_{1}}\left|\left\langle\frac{\mathrm{d} m}{\mathrm{~d} t}, D m\right\rangle\right|+\int_{T_{1}}^{T}\left|\left\langle\frac{\mathrm{d} m}{\mathrm{~d} t}, D m\right\rangle\right|\right) \\
& \geqslant 2 C(\alpha)\left(\left|\int_{0}^{T_{1}}\left\langle\frac{\mathrm{d} m}{\mathrm{~d} t}, D m\right\rangle\right|+\left|\int_{T_{1}}^{T}\left\langle\frac{\mathrm{d} m}{\mathrm{~d} t}, D m\right\rangle\right|\right) \\
& =4 C(\alpha)\left[\left\langle D m\left(T_{1}\right), m\left(T_{1}\right\rangle-\alpha_{1}\right]\right. \\
& \geqslant 4 C(\alpha)\left(\alpha_{2}-\alpha_{1}\right) .
\end{aligned}
$$

This holds for every $m \in V_{T}$, which justifies (2.13).

\section{PDE WEAK SOLUTIONS}

\subsection{Existence of 3D global weak solutions}

Weak solutions for Landau-Lifschitz equations have been proven to exist in $[1,15,18]$ although either without a possibly variable in time external field or without the stray-field. We here follow the strategy of [1], and show necessary adaptations to our case.

Definition 3.1. Let $m_{0} \in H^{1}\left(\Omega, S^{2}\right)$ and $H_{\mathrm{ext}} \in L_{\text {loc }}^{1}\left(\mathbb{R}, \mathbb{R}^{3}\right)$. A function $m$ is a weak solution of (1.9) if

- for every $T>0, m \in H^{1}\left(Q_{T}, S^{2}\right)$;

- for every $T>0$, for every $\Phi \in H^{1}\left(Q_{T}, \mathbb{R}^{3}\right)$, there holds

$$
\begin{aligned}
\int_{Q_{T}}\left\langle\frac{\partial m}{\partial t}, \Phi\right\rangle-\alpha\left\langle m \wedge \frac{\partial m}{\partial t}, \Phi\right\rangle \mathrm{d} x \mathrm{~d} t= \\
\quad-\left(1+\alpha^{2}\right) \int_{Q_{T}}-\sum_{j=1}^{3}\left\langle m \wedge \frac{\partial m}{\partial x_{j}}, \frac{\partial \Phi}{\partial x_{j}}\right\rangle+\left\langle m \wedge\left(H_{d}(m)+H_{\text {ext }}\right), \Phi\right\rangle \mathrm{d} x \mathrm{~d} t
\end{aligned}
$$

- $m(0, x)=m_{0}(x)$ in the trace sense;

- for almost every $T>0$,

$$
\mathcal{E}(m(T))+\frac{\alpha}{1+\alpha^{2}} \int_{0}^{T}\left\|\frac{\partial m}{\partial t}(t)\right\|_{L^{2}(\Omega)}^{2} \mathrm{~d} t \leqslant \mathcal{E}\left(m_{0}\right)+\int_{0}^{T} \int_{\Omega}\left\langle H_{\text {ext }}, \frac{\partial m}{\partial t}\right\rangle,
$$

where $\mathcal{E}(m)$ is the micromagnetic energy defined by

$$
\mathcal{E}(m):=\frac{1}{2} \int_{\Omega}|\nabla m|^{2}+\frac{1}{2} \int_{\mathbb{R}^{3}}\left|H_{d}(m)\right|^{2} .
$$

Let us now state the main result of this section.

Theorem 3.1. Let $m_{0} \in H^{1}\left(\Omega, S^{2}\right)$ and $H_{\mathrm{ext}} \in L_{\mathrm{loc}}^{2}\left(\mathbb{R}_{+}, \mathbb{R}^{3}\right)$. There exists a weak solution of (1.9).

Remark 3.1. Notice that such weak solutions may not be unique (see [1], Thm. 1.6). 
The proof of Theorem 3.1 readily follows the arguments of [1], Theorem 1.5. We write a complete proof in order to precise the necessary adaptations, due to the presence of $H_{d}(m)$ and $H_{\text {ext }}$ in (1.9).

Proof of Theorem 3.1. First, following [1], we construct, through a Galerkin method, weak solutions to the penalized system

$$
\left(P^{k}\right)\left\{\begin{array}{l}
\alpha \frac{\partial m^{k}}{\partial t}+m^{k} \wedge \frac{\partial m^{k}}{\partial t}=\left(1+\alpha^{2}\right)\left[\Delta m^{k}+H_{d}\left(m^{k}\right)+H_{\mathrm{ext}}-k\left(\left|m^{k}\right|^{2}-1\right) m^{k}\right] \text { in } \Omega \\
m^{k}(0)=m_{0}, \\
\frac{\partial m^{k}}{\partial \nu}(t, x)=0, x \in \partial \Omega,
\end{array}\right.
$$

for $k \in \mathbb{R}_{+}^{*}$, where the constraint $|m|=1$ is relaxed.

Let $\left(\varphi_{j}\right)_{j \in \mathbb{N}}$ be an orthonormal basis of $L^{2}(\Omega, \mathbb{R})$ consisting of eigenvectors of the Laplace operator with Neumann boundary conditions,

$$
\left\{\begin{array}{l}
-\Delta \varphi_{j}=\lambda_{j} \varphi_{j}, \text { in } \Omega \\
\frac{\partial \varphi_{j}}{\partial \nu}=0, \text { on } \partial \Omega
\end{array}\right.
$$

where $\left(\lambda_{j}\right)_{j \in \mathbb{N}}$ is a non decreasing sequence. Let $n \in \mathbb{N}^{*}$. We are looking for approximate solutions

$$
m_{n}(t, x)=\sum_{j=0}^{n-1} y_{j}(t) \varphi_{j}(x)
$$

where

- for every $T>0, y_{j} \in H^{1}\left((0, T), \mathbb{R}^{3}\right)$;

- for almost every $t \in(0,+\infty)$, for every $l \in\{0, \ldots, n-1\}$,

$$
\left\{\begin{array}{l}
\int_{\Omega}\left\{\frac{1}{\alpha^{2}+1}\left(\alpha \frac{\partial m_{n}}{\partial t}+m_{n} \wedge \frac{\partial m_{n}}{\partial t}\right)\right. \\
\left.\quad-\left[\Delta m_{n}+H_{d}\left(m_{n}\right)+H_{\text {ext }}-k\left(\left|m_{n}\right|^{2}-1\right) m_{n}\right]\right\}(t, x) \varphi_{l}(x) \mathrm{d} x=0 \\
\int_{\Omega}\left[m_{n}(0, x)-m_{0}(x)\right] \varphi_{l}(x) \mathrm{d} x=0 .
\end{array}\right.
$$

These relations produce a differential system that can be written as

$$
\left\{\begin{array}{l}
\frac{\partial Y}{\partial t}-A(Y) \frac{\partial Y}{\partial t}=F(Y)+B(t), \text { for almost every } t \in(0,+\infty) \\
Y(0)=Y_{0}
\end{array}\right.
$$

where $Y(t):=\left(\left(y_{0}(t), \ldots, y_{n-1}(t)\right)^{\tau} \in \mathbb{R}^{3 n}\right), A(Y)$ is a $3 n \times 3 n$ skew-symmetric matrix (thus $I-A(Y)$ is always invertible), $F: \mathbb{R}^{3 n} \rightarrow \mathbb{R}^{3 n}$ is a locally Lipschitz nonlinear map and $B(t):=\left(B_{0}(t), \ldots, B_{n-1}(t)\right)^{\tau} \in \mathbb{R}^{3 n}$ has components

$$
B_{j}:=\frac{\left(1+\alpha^{2}\right)}{\alpha} H_{\mathrm{ext}}(t) \delta_{j, 0}
$$

A fixed point argument gives the existence of $\tilde{T}_{n}>0$ and the existence and uniqueness of $Y \in C^{0}\left(\left(0, \tilde{T}_{n}\right), \mathbb{R}^{3 n}\right)$ such that, for every $t \in\left(0, \tilde{T}_{n}\right)$,

$$
Y(t)=Y_{0}+\int_{0}^{t}(I-A(Y(s)))^{-1}[F(Y(s))+B(s)] \mathrm{d} s,
$$

moreover, $Y \in H^{1}\left(\left(0, \tilde{T}_{n}\right), \mathbb{R}^{3 n}\right)$ and $(3.5)$ is satisfied. 
Remark 3.2. If $H_{\text {ext }} \in C^{0}\left(\mathbb{R}_{+}, \mathbb{R}^{3}\right)$ then $Y \in C^{1}\left(\left(0, T_{n}\right), \mathbb{R}^{3 n}\right)$, the first equality of (3.5) holds for every $t \in\left(0, T_{n}\right)$ and the proof may be finished exactly as in [1]. When $H_{\mathrm{ext}} \in L_{\mathrm{loc}}^{2}\left(\mathbb{R}_{+}, \mathbb{R}^{3}\right)$, the first equality of (3.5) may only hold a.e., this changes a few details at the end of the proof.

Let $T_{n} \in(0,+\infty]$ and $Y \in C^{0}\left(\left(0, T_{n}\right), \mathbb{R}^{3 n}\right)$ be a maximal solution. In order to prove that $T_{n}=+\infty$, we proceed by contradiction, assuming that $T_{n}<+\infty$ and $Y(t)$ is not bounded when $t \rightarrow T_{n}$. Noticing that

$$
\|Y(t)\|^{2}=\int_{\Omega}\left|m_{n}(t, x)\right|^{2} \mathrm{~d} x
$$

we provide estimates on $m_{n}$ showing that $m_{n} \in L^{\infty}\left(\left(0, T_{n}\right), L^{2}(\Omega)\right)$, which gives the contradiction.

Multiplying the first equation of (3.4) by $\frac{\mathrm{d} y_{l}}{\mathrm{~d} t}(t)$ (which is finite for almost every $t \in\left(0, T_{n}\right)$ ) and summing for $l=0, \ldots, n-1$, we find, for almost every $t \in\left(0, T_{n}\right)$,

$$
\begin{aligned}
\frac{\alpha}{\alpha^{2}+1} \int_{\Omega}\left|\frac{\partial m_{n}}{\partial t}\right|^{2}=-\frac{\mathrm{d}}{\mathrm{d} t} & {\left[\frac{1}{2} \int_{\Omega}\left(\left|\nabla m_{n}\right|^{2}+\frac{k}{2}\left(\left|m_{n}\right|^{2}-1\right)^{2}\right)+\frac{1}{2} \int_{\mathbb{R}^{3}}\left|H_{d}\left(m_{n}\right)\right|^{2}\right] } \\
& +\left\langle H_{\text {ext }}, \int_{\Omega} \frac{\partial m_{n}}{\partial t}\right\rangle .
\end{aligned}
$$

Integrating this relation between 0 and $t$ leads to

$$
\mathcal{E}_{k}\left(m_{n}(t)\right)+\frac{\alpha}{\alpha^{2}+1} \int_{0}^{t} \int_{\Omega}\left|\frac{\partial m_{n}}{\partial t}\right|^{2} \leqslant \mathcal{E}_{k}\left(m_{n 0}\right)+\int_{0}^{t}\left\langle H_{\mathrm{ext}}, \int_{\Omega} \frac{\partial m_{n}}{\partial t}\right\rangle
$$

for almost every $t \in\left(0, T_{n}\right)$, where $m_{n 0}(x):=m_{n}(0, x)$, and

$$
\mathcal{E}_{k}(m):=\frac{1}{2} \int_{\Omega}\left(|\nabla m|^{2}+\frac{k}{2}\left(|m|^{2}-1\right)^{2}\right)+\frac{1}{2} \int_{\mathbb{R}^{3}}\left|H_{d}(m)\right|^{2} .
$$

Using the inequality

$$
\left\langle H_{\mathrm{ext}}, \frac{\partial m_{n}}{\partial t}\right\rangle \leqslant \frac{\alpha^{2}+1}{2 \alpha}\left|H_{\mathrm{ext}}\right|^{2}+\frac{\alpha}{2\left(\alpha^{2}+1\right)}\left|\frac{\partial m_{n}}{\partial t}\right|^{2}
$$

we deduce that

$$
\mathcal{E}_{k}\left(m_{n}(t)\right)+\frac{\alpha}{2\left(\alpha^{2}+1\right)} \int_{0}^{t} \int_{\Omega}\left|\frac{\partial m_{n}}{\partial t}\right|^{2} \leqslant \mathcal{E}_{k}\left(m_{n 0}\right)+|\Omega| \frac{\alpha^{2}+1}{2 \alpha} \int_{0}^{t}\left|H_{\mathrm{ext}}\right|^{2} .
$$

Moreover, since for every $t \in\left(0, T_{n}\right)$,

$$
\int_{\Omega}\left|m_{n}(t, x)\right|^{2} \mathrm{~d} x \leqslant\left(|\Omega| \int_{\Omega}\left(\left|m_{n}(t, x)\right|^{2}-1\right)^{2}\right)^{1 / 2}+|\Omega|,
$$

then (3.8) provides a bound of the right-hand side, and we get that $m_{n} \in L^{\infty}\left(\left(0, T_{n}\right), L^{2}(\Omega)\right)$, which gives the contradiction. Finally, the maximal solution $Y(t)$ is defined for every $t \in(0,+\infty)$, and for every $T>0$, $m_{n} \in H^{1}\left(Q_{T}\right)$. 
Let $T>0$. Since $H^{1}(\Omega)$ is embedded into $L^{4}(\Omega)$, the right hand side of $(3.8)$, is bounded uniformly with respect to $n \in \mathbb{N}^{*}$ and $t \in[0, T]$ by

$$
\mathcal{R}_{k}:=C_{k}\left\|m_{0}\right\|_{H^{1}(\Omega)}+|\Omega| \frac{\alpha^{2}+1}{2 \alpha} \int_{0}^{T}\left|H_{\mathrm{ext}}\right|^{2}
$$

where $C_{k}=C(\Omega, k)$.

The inequality (3.8) shows that $\left(\left|m_{n}\right|^{2}-1\right), \frac{\partial m_{n}}{\partial t}$ and $\nabla m_{n}$ are bounded in $L^{2}\left(Q_{T}, \mathbb{R}^{3}\right)$, indeed,

$$
\begin{aligned}
& \int_{0}^{T} \mathcal{E}_{k}\left(m_{n}(t)\right) \mathrm{d} t \leqslant T \mathcal{R}_{k}, \\
& \frac{\alpha}{2\left(\alpha^{2}+1\right)} \int_{0}^{T} \int_{\Omega}\left|\frac{\partial m_{n}}{\partial t}(t, x)\right|^{2} \mathrm{~d} x \mathrm{~d} t \leqslant \mathcal{R}_{k} .
\end{aligned}
$$

Thus $m_{n}$ is also bounded in $H^{1}\left(Q_{T}\right)$ (the uniform $L^{2}$ bound is given by (3.9)) and there exists $m^{k} \in H^{1}\left(Q_{T}\right)$ such that, up to the extraction of a subsequence,

$$
\begin{aligned}
& m_{n} \rightarrow m^{k} \text { weakly in } H^{1}\left(Q_{T}\right), \\
& m_{n} \rightarrow m^{k} \text { strongly in } L^{2}\left(Q_{T}\right), \\
& \left|m_{n}\right|^{2}-1 \rightarrow\left|m^{k}\right|^{2}-1 \text { weakly in } L^{2}\left(Q_{T}\right) .
\end{aligned}
$$

Passing to the limit $(n \rightarrow+\infty)$ in $(3.7)$ gives $\left(\right.$ since $\left.\mathcal{E}_{k}\left(m_{0}\right)=\mathcal{E}_{0}\left(m_{0}\right)\right)$

$$
\mathcal{E}_{k}\left(m^{k}(t)\right)+\frac{\alpha}{\alpha^{2}+1} \int_{0}^{t} \int_{\Omega}\left|\frac{\partial m^{k}}{\partial t}\right|^{2} \leqslant \mathcal{E}_{0}\left(m_{0}\right)+\int_{0}^{t}\left\langle H_{\mathrm{ext}}, \int_{\Omega} \frac{\partial m^{k}}{\partial t}\right\rangle
$$

for almost every $t \in[0, T]$.

Now, let $N \in \mathbb{N}^{*}$ and $\varphi=\sum_{j=0}^{N-1} \alpha_{j}(t) \varphi_{j}$, where $\forall j \in\{0, \ldots, N-1\}, \alpha_{j} \in \mathcal{C}^{\infty}\left([0, T], \mathbb{R}^{3}\right)$. For every $n \in \mathbb{N}^{*}$ with $n \geqslant N$, we have

$$
\begin{aligned}
\int_{Q_{T}}\left\langle\alpha \frac{\partial m_{n}}{\partial t}+m_{n} \wedge \frac{\partial m_{n}}{\partial t}, \varphi\right\rangle= & -\left(\alpha^{2}+1\right) \int_{Q_{T}}\left\langle\nabla m_{n}, \nabla \varphi\right\rangle \\
& +\left(\alpha^{2}+1\right) \int_{Q_{T}}\left\langle H_{d}\left(m_{n}\right)+H_{\mathrm{ext}}-k\left(\left|m_{n}\right|^{2}-1\right) m_{n}, \varphi\right\rangle,
\end{aligned}
$$

which gives, passing to the limit $n \rightarrow+\infty$

$$
\begin{aligned}
\int_{Q_{T}}\left\langle\alpha \frac{\partial m^{k}}{\partial t}+m^{k} \wedge \frac{\partial m^{k}}{\partial t}, \varphi\right\rangle= & -\left(\alpha^{2}+1\right) \int_{Q_{T}}\left\langle\nabla m^{k}, \nabla \varphi\right\rangle \\
& +\left(\alpha^{2}+1\right) \int_{Q_{T}}\left\langle H_{d}\left(m^{k}\right)+H_{\mathrm{ext}}-k\left(\left|m^{k}\right|^{2}-1\right) m^{k}, \varphi\right\rangle .
\end{aligned}
$$

Indeed, thanks to (1.8), we have

$$
\begin{aligned}
\left\|H_{d}\left(m_{n}-m^{k}\right)\right\|_{L^{2}\left(Q_{T}\right)} & \leqslant\left\|m_{n}-m^{k}\right\|_{L^{2}\left(Q_{T}\right)} \\
& \rightarrow 0 \text { when } n \rightarrow+\infty .
\end{aligned}
$$

By density, (3.12) also holds for every $\varphi \in H^{1}\left(Q_{T}, \mathbb{R}^{3}\right)$. 
From (3.11), we get that $\nabla m^{k}, H_{d}\left(m^{k}\right), \sqrt{k}\left(\left|m^{k}\right|^{2}-1\right), \frac{\partial m^{k}}{\partial t}$ are bounded in $L^{2}\left(Q_{T}\right)$, and therefore, there exists $m \in H^{1}\left(Q_{T}\right)$ such that, up to the extraction of a suitable subsequence,

$$
\begin{aligned}
& m^{k} \rightarrow m \text { weakly in } H^{1}\left(Q_{T}\right), \\
& m^{k} \rightarrow m \text { strongly in } L^{2}\left(Q_{T}\right) \\
& \left|m^{k}\right|^{2}-1 \rightarrow 0 \text { strongly in } L^{2}\left(Q_{T}\right)
\end{aligned}
$$

We hence first obtain, $|m|=1$ a.e. on $Q_{T}$, and in order to pass to the limit $k \rightarrow+\infty$ in (3.12), we take $\Phi \in \mathcal{C}^{\infty}\left(Q_{T}\right)$, and test (3.12) with $\varphi(t, x):=m^{k} \wedge \Phi \in H^{1}\left(Q_{T}\right)$. We get

$$
\begin{aligned}
& \int_{Q_{T}} \alpha\left\langle\frac{\partial m^{k}}{\partial t}\right.\left.\wedge m^{k}, \Phi\right\rangle-\left\langle m^{k}, \frac{\partial m^{k}}{\partial t}\right\rangle\left\langle m^{k}, \Phi\right\rangle+\left|m^{k}\right|^{2}\left\langle\frac{\partial m^{k}}{\partial t}, \Phi\right\rangle= \\
&\left(\alpha^{2}+1\right) \int_{Q_{T}}-\left\langle\nabla m^{k} \wedge m^{k}, \nabla \Phi\right\rangle+\left\langle\left(H_{d}\left(m^{k}\right)+H_{\text {ext }}\right) \wedge m^{k}, \Phi\right\rangle .
\end{aligned}
$$

All the terms pass to the limit easily but two, namely, $\int_{Q_{T}}\left\langle m^{k}, \frac{\partial m^{k}}{\partial t}\right\rangle\left\langle m^{k}, \Phi\right\rangle$ and $\int_{Q_{T}}\left|m^{k}\right|^{2}\left\langle\frac{\partial m^{k}}{\partial t}, \Phi\right\rangle$. We decompose this latter term as

$$
\begin{aligned}
\int_{Q_{T}}\left|m^{k}\right|^{2}\left\langle\frac{\partial m^{k}}{\partial t}, \Phi\right\rangle & =\int_{Q_{T}}\left(\left|m^{k}\right|^{2}-1\right)\left\langle\frac{\partial m^{k}}{\partial t}, \Phi\right\rangle+\int_{Q_{T}}\left\langle\frac{\partial m^{k}}{\partial t}, \Phi\right\rangle \\
& \rightarrow \int_{Q_{T}}\left\langle\frac{\partial m}{\partial t}, \Phi\right\rangle
\end{aligned}
$$

when $k$ tends to infinity, from (3.13).

For the other term, we perform an integration by parts

$$
\begin{aligned}
-\int_{Q_{T}}\left\langle m^{k}, \frac{\partial m^{k}}{\partial t}\right\rangle\left\langle m^{k}, \Phi\right\rangle & =-\int_{Q_{T}} \frac{1}{2} \frac{\mathrm{d}}{\mathrm{d} t}\left[\left|m^{k}\right|^{2}-1\right]\left\langle m^{k}, \Phi\right\rangle \\
& =\frac{1}{2} \int_{Q_{T}}\left[\left|m^{k}\right|^{2}-1\right] \frac{\mathrm{d}}{\mathrm{d} t}\left[\left\langle m^{k}, \Phi\right\rangle\right] \\
& \rightarrow 0 \text { as } k \rightarrow+\infty .
\end{aligned}
$$

Remark 3.3. This step is different from [1]: we do not need here that $\left|m^{k}\right| \leqslant 1$ a.e. which was proved with a Stampacchia argument in [1].

Eventually, in order to show that $m$ satisfies the energy inequality (3.2), we just pass to the weak $H^{1}\left(Q_{T}\right)$ limit in (3.11).

\subsection{Convergence of weak solutions to ODE solutions when the size of the domain goes to zero}

Let $\Omega$ be a bounded open subset of $\mathbb{R}^{2}$ or $\mathbb{R}^{3}$ such that $|\Omega|=1$. In this section, we consider the weak solutions of the Landau-Lifschitz PDE on the domain $\Omega_{\lambda}:=\sqrt{\lambda} \Omega$, when $\lambda \rightarrow 0, \lambda>0$. A change of space and time variables shows that it is equivalent to study the weak solutions of the following Landau-Lifschitz PDE 
on the fixed domain $\Omega$,

$$
\left\{\begin{array}{l}
\frac{\partial m}{\partial t}=\alpha\left[H_{\lambda}(m)-\left\langle H_{\lambda}(m), m\right\rangle m\right]-m \wedge H_{\lambda}(m), \quad x \in \Omega, \quad t \in(0, T) \\
\frac{\partial m}{\partial \nu}(t, x)=0, \quad x \in \partial \Omega, \quad t \in(0, T) \\
m(0, x)=m_{0}(x), \quad x \in \Omega
\end{array}\right.
$$

with an effective magnetic field

$$
H_{\lambda}(m):=\frac{\Delta m}{\lambda}+H_{d}(m)+H_{\mathrm{ext}}
$$

associated to the micromagnetic energy

$$
\mathcal{E}_{\lambda}(m):=\int_{\Omega} \frac{1}{2 \lambda}|\nabla m|^{2}+\frac{1}{2} \int_{\mathbb{R}^{3}}\left|H_{d}(m)\right|^{2} .
$$

When the domain is small $(\lambda<<1)$, non constant in space magnetizations are penalized. Therefore it is expected that solutions of (3.15), (3.16) should tend to the solutions of the ODE (2.1) with $D$ defined by

$$
D \widetilde{m}:=-\frac{1}{|\Omega|} \int_{\Omega} H_{d}\left(\widetilde{m} \chi_{\Omega}\right), \quad \forall \widetilde{m} \in S^{2},
$$

where $\chi_{\Omega}$ is the characteristic function of $\Omega$. This is precisely the purpose of this section. We also quote the paper by DeSimone [10] in which the same kind of result is shown but for static problems, using $\Gamma$-convergence theory.

The convergence result proved in this section shows that the external magnetic field found in Section 2 allows an approximate switching for the PDE solutions, on any sufficiently small domain, in the very general sense of weak solutions.

Proposition 3.1. Let $\Omega$ be a bounded open subset of $\mathbb{R}^{2}$ or $\mathbb{R}^{3}, \alpha>0, H_{\mathrm{ext}} \in L_{\mathrm{loc}}^{2}\left(\mathbb{R}_{+}, \mathbb{R}^{3}\right), \bar{m} \in S^{2}$. Let $\left(m_{\lambda 0}\right)_{\lambda>0}$ be a sequence of $H^{2}\left(\Omega, S^{2}\right)$ such that $\frac{\partial m_{\lambda 0}}{\partial \nu} \equiv 0$ on $\partial \Omega$ for every $\lambda>0$,

$$
m_{\lambda 0 \sharp} \rightarrow \bar{m} \text { and } \int_{\Omega}\left|\nabla m_{\lambda 0}\right|^{2}=o(\sqrt{\lambda}) \text { when } \lambda \rightarrow 0 .
$$

Let $m_{\lambda}$ be a weak solution of (3.15) such that $m_{\lambda}(0)=m_{\lambda 0}$ and $m_{\mathrm{ref}}$ be the solution of (2.1) with initial data $m_{0}=\bar{m}$. Then, for every $T>0$,

$$
\left\|m_{\lambda}-m_{\mathrm{ref}}\right\|_{C^{0}\left([0, T], H^{1}(\Omega)\right)} \rightarrow 0 \text { when } \lambda \rightarrow 0 .
$$

Remark 3.4. The assumption (3.18) is not restrictive as the minimizers of $\mathcal{E}_{\lambda}$ do satisfy it.

Proof of Proposition 3.1. The proof follows 3 steps.

First step: Bounds on $\nabla m_{\lambda}$ and $\frac{\partial m_{\lambda}}{\partial t}$. We know (from the definition of 'weak solution') that

$$
\mathcal{E}_{\lambda}\left(m_{\lambda}(t)\right)+\frac{\alpha}{2\left(\alpha^{2}+1\right)} \int_{0}^{t}\left\|\frac{\partial m_{\lambda}}{\partial t}\right\|_{L^{2}}^{2} \leqslant \mathcal{E}_{\lambda}\left(m_{\lambda}(0)\right)+|\Omega| \frac{\left(\alpha^{2}+1\right)}{2 \alpha} \int_{0}^{t}\left|H_{\mathrm{ext}}\right|^{2}
$$

with $\mathcal{E}_{\lambda}$ defined by (3.17). Thus, we have the following bounds

$$
\left\|\nabla m_{\lambda}\right\|_{C^{0}\left([0, T], L^{2}\right)}^{2} \leqslant 2 \lambda\left(\mathcal{E}_{\lambda}\left(m_{\lambda}(0)\right)+|\Omega| \frac{\alpha^{2}+1}{2 \alpha} \int_{0}^{T}\left|H_{\mathrm{ext}}\right|^{2}\right),
$$


and

$$
\int_{0}^{T}\left\|\frac{\partial m_{\lambda}}{\partial t}\right\|_{L^{2}}^{2} \leqslant \frac{2\left(\alpha^{2}+1\right)}{\alpha}\left(\mathcal{E}_{\lambda}\left(m_{\lambda}(0)\right)+|\Omega| \frac{\alpha^{2}+1}{2 \alpha} \int_{0}^{T}\left|H_{\mathrm{ext}}\right|^{2}\right) .
$$

Second step: Study of $m_{\sharp}$. Taking in (3.1) a test function $\Phi$ constant in space (but not in time) leads to an equation for $m_{\lambda \sharp}$

$$
\frac{\mathrm{d} m_{\lambda \sharp}}{\mathrm{d} t}-\alpha m_{\lambda \sharp} \wedge \frac{\mathrm{d} m_{\lambda \sharp}}{\mathrm{d} t}=-\left(1+\alpha^{2}\right) m_{\lambda \sharp} \wedge H_{0}\left(m_{\lambda \sharp}\right)+f_{\lambda}(t),
$$

where from the definition $(2.2)$ of $H_{0}$

$$
f_{\lambda}(t):=\frac{1}{|\Omega|} \int_{\Omega} \alpha\left(m_{\lambda}-m_{\lambda \sharp}\right) \wedge \frac{\partial m_{\lambda}}{\partial t}-\frac{\left(1+\alpha^{2}\right)}{|\Omega|} \int_{\Omega} m_{\lambda} \wedge\left(H_{d}\left(m_{\lambda}\right)+D m_{\lambda \sharp}\right) .
$$

Now, since $D m_{\lambda \sharp \chi_{\Omega}}=-\frac{1}{|\Omega|} \int_{\Omega} H_{d}\left(m_{\lambda \sharp}\right)$, we may write the latter term as

$$
\int_{\Omega} m_{\lambda} \wedge\left(H_{d}\left(m_{\lambda}\right)+D m_{\lambda \sharp}\right)=\int_{\Omega} m_{\lambda} \wedge H_{d}\left(m_{\lambda}-m_{\lambda \sharp \chi_{\Omega}}\right)+\left(m_{\lambda}-m_{\lambda \sharp}\right) \wedge\left(H_{d}\left(m_{\lambda \sharp} \chi_{\Omega}\right)+D m_{\lambda \sharp}\right)
$$

from which we can bound

$$
\left|f_{\lambda}(t)\right| \leq \frac{\alpha}{|\Omega|}\left\|m_{\lambda}-m_{\lambda \sharp}\right\|_{L^{2}}\left\|\frac{\partial m_{\lambda}}{\partial t}\right\|_{L^{2}}+\frac{\left(1+\alpha^{2}\right)}{\sqrt{|\Omega|}}\left(\left\|H_{d}\left(m_{\lambda}-m_{\lambda \sharp} \chi_{\Omega}\right)\right\|_{L^{2}}+2\left\|m_{\lambda}-m_{\lambda \sharp}\right\|_{L^{2}}\right) .
$$

This implies, thanks to (3.19), (3.20) and Poincaré inequality that there exists $C_{2}=C_{2}(\Omega, \alpha, T)>0$ such that

$$
\int_{0}^{T}\left|f_{\lambda}(t)\right| \mathrm{d} t \leqslant C_{2}[B(\lambda)+\sqrt{B(\lambda)}] \sqrt{\lambda}
$$

where

$$
B(\lambda):=\mathcal{E}_{\lambda}\left(m_{\lambda}(0)\right)+|\Omega| \frac{\alpha^{2}+1}{2 \alpha} \int_{0}^{T}\left|H_{\mathrm{ext}}\right|^{2} .
$$

We notice that from (3.21) we can deduce

$$
\left\langle m_{\lambda \sharp}, \frac{\mathrm{d} m_{\lambda \sharp}}{\mathrm{d} t}\right\rangle=\left\langle m_{\lambda \sharp}, f_{\lambda}(t)\right\rangle
$$

from which we get

$$
\begin{aligned}
\frac{\mathrm{d} m_{\lambda \sharp}}{\mathrm{d} t} & =F\left(m_{\lambda \sharp}\right)+\frac{1}{1+\alpha^{2}\left|m_{\lambda \sharp}\right|^{2}}\left(\alpha^{2}\left\langle m_{\lambda \sharp}, f_{\lambda}(t)\right\rangle m_{\lambda \sharp}+f_{\lambda}(t)+\alpha m_{\lambda \sharp} \wedge f_{\lambda}(t)\right), \\
\frac{\mathrm{d} m_{\mathrm{ref}}}{\mathrm{d} t} & =F\left(m_{\mathrm{ref}}\right),
\end{aligned}
$$

where

$$
\begin{aligned}
F: \mathbb{R}^{3} & \rightarrow \mathbb{R}^{3} \\
m & \mapsto-\frac{1+\alpha^{2}}{1+\alpha^{2}|m|^{2}}\left(m \wedge H_{0}(m)+\alpha m \wedge\left(m \wedge H_{0}(m)\right)\right)
\end{aligned}
$$

satisfies

$$
\forall m_{1}, m_{2} \in B_{\mathbb{R}^{3}}(0,1),\left|F\left(m_{1}\right)-F\left(m_{2}\right)\right| \leqslant C_{3}\left(1+\left|H_{\text {ext }}(t)\right|\right)\left|m_{1}-m_{2}\right|,
$$


for some constant $C_{3}=C_{3}(\alpha, D)$. Using Gronwall lemma and (3.22), we get

$$
\left|m_{\lambda \sharp}-m_{\mathrm{ref}}\right|_{C^{0}\left([0, T], \mathbb{R}^{3}\right)} \leqslant\left(\left|m_{\lambda 0 \sharp}-\bar{m}\right|+C_{2} \sqrt{\lambda}[B+\sqrt{B}]\right) \mathrm{e}^{C_{3}\left(T+\int_{0}^{T}\left|H_{\text {ext }}\right|\right)} .
$$

Third step: Conclusion. Noticing that

$$
\left\|m_{\lambda}-m_{\mathrm{ref}} \chi_{\Omega}\right\|_{L^{2}}^{2}=2|\Omega|\left(1-\left\langle m_{\lambda \sharp}, m_{\mathrm{ref}}\right\rangle\right)=2|\Omega|\left\langle m_{\mathrm{ref}}, m_{\mathrm{ref}}-m_{\lambda \sharp}\right\rangle,
$$

we get

$$
\left\|m_{\lambda}-m_{\mathrm{ref}} \chi_{\Omega}\right\|_{C^{0}\left([0, T], H^{1}\right)}^{2} \leqslant 2 \lambda B+2|\Omega|\left(\left|m_{\lambda 0 \sharp}-\bar{m}\right|+C_{2} \sqrt{\lambda}[B+\sqrt{B}]\right) \mathrm{e}^{C_{3}\left(T+\int_{0}^{T}\left|H_{\text {ext }}\right|\right)}
$$

and the conclusion comes from (3.18).

It is well known (see [1] for examples) that, for $\lambda>0$ fixed, there may not be uniqueness for the weak solutions of $(3.15),(3.16)$. However, all these weak solutions converge in $C^{0}\left([0, T], H^{1}(\Omega)\right)$ to the same function $m_{\text {ref }}$, when $\lambda \rightarrow 0$.

Although restricted to approximate controllability, the preceding result is very general in the sense that it applies to a wide class of solutions to Landau-Lifschitz equations. To go further and obtain stronger results (like stabilization and convergence to minimizers), we need stronger solutions. This is precisely the aim of the following sections.

\section{Global PDE smooth solutions}

In this section, we investigate the existence and uniqueness of global smooth solutions of the Landau-Lifschitz equation (1.9) on the domain $\Omega_{\lambda}:=\sqrt{\lambda} \Omega$, with $\lambda>0$. As already explained, it is equivalent to study (3.15) and (3.16). First, we show existence and uniqueness of local (in time) smooth solutions.

Theorem 4.1. Let $\Omega$ be a bounded regular open subset of $\mathbb{R}^{2}$ or $\mathbb{R}^{3}, \alpha>0, \lambda>0, H_{\text {ext }} \in C^{0}\left(\mathbb{R}_{+}, \mathbb{R}^{3}\right)$ and $m_{0} \in H^{2}\left(\Omega, S^{2}\right)$ be such that $\frac{\partial m_{0}}{\partial \nu}=0$ on $\partial \Omega$. There exist a time $T^{*}=T^{*}\left(\Omega, \alpha, \lambda,\left\|m_{0}\right\|_{H^{2}(\Omega)},\left\|H_{\mathrm{ext}}\right\|_{L^{\infty}}\right)$ and a unique

$$
m \in C^{0}\left([0, T], H^{2}\left(\Omega, S^{2}\right)\right) \cap H^{1}\left((0, T), H^{1}\left(\Omega, S^{2}\right)\right) \cap L^{2}\left((0, T), H^{3}\left(\Omega, S^{2}\right)\right),
$$

for all $T \in\left(0, T^{*}\right)$, satisfying (3.15) and (3.16). Moreover, such regular solutions depend continuously on $m_{0}$ for the topology $C^{0}\left([0, T], H^{2}\left(\Omega, S^{2}\right)\right)$.

In the $2 \mathrm{D}$ case, this result can be improved since global existence holds for small $\lambda$ (i.e. small domains $\Omega_{\lambda}=\sqrt{\lambda} \Omega$ ), with initial conditions $m_{0}$ in a $H^{1}$-neighborhood of constants, and for all (bounded and regular) domains $\Omega$.

Theorem 4.2. Let $\Omega$ be a bounded open subset of $\mathbb{R}^{2}, \alpha>0, \lambda>0, H_{\mathrm{ext}} \in L^{\infty}\left(\mathbb{R}_{+}, \mathbb{R}^{3}\right)$ with $\dot{H}_{\mathrm{ext}} \in L^{1}\left(\mathbb{R}_{+}, \mathbb{R}^{3}\right)$ and $C^{*}(\Omega)$ be the smallest constant $C$ such that (4.13) holds. For every $m_{0} \in H^{2}\left(\Omega, S^{2}\right)$ such that $\frac{\partial m_{0}}{\partial \nu}=0$ on $\partial \Omega$ and

$$
\int_{\Omega}\left|\nabla m_{0}\right|^{2}+\lambda\left(\int_{\Omega}\left|H_{d}\left(m_{0}\right)\right|^{2}+4\left\|H_{\mathrm{ext}}\right\|_{L^{\infty}\left(\mathbb{R}_{+}, \mathbb{R}^{3}\right)}+2\left\|\dot{H}_{\mathrm{ext}}\right\|_{L^{1}\left(\mathbb{R}_{+}, \mathbb{R}^{3}\right)}\right)<\frac{1}{C^{*}(\Omega)}
$$

the smooth solution of (3.15) exists on $\mathbb{R}_{+}$.

In the $3 \mathrm{D}$ case, the result of Theorem 4.1 can also be improved, when $\Omega$ is an ellipsoidal domain. We get in that case global existence of smooth solutions for small $\lambda$ (i.e. small ellipsoids $\Omega_{\lambda}=\sqrt{\lambda} \Omega$ ), and for initial conditions $m_{0}$ in a $H^{2}$-neighborhood of constants. 
Theorem 4.3. Let $\Omega$ be a $3 D$ ellipsoid domain. There exists $C^{* *}(\Omega)>0$ such that, for every $\alpha>0$, for every $\lambda \in(0,1)$, for every $H_{\mathrm{ext}} \in C^{0} \cap L^{\infty}\left(\mathbb{R}_{+}, \mathbb{R}^{3}\right)$, for every $m_{0} \in H^{2}\left(\Omega, S^{2}\right)$ with $\frac{\partial m_{0}}{\partial \nu}=0$ on $\partial \Omega$ that satisfy

$$
\begin{gathered}
C^{* *}(\alpha+1)\left(1+\left\|H_{\text {ext }}\right\|_{L^{\infty}\left(\mathbb{R}_{+}\right)}\right) \leqslant \frac{\alpha}{\lambda}, \\
C^{* *}(\alpha+1)\left[\left\|\Delta m_{0}\right\|_{L^{2}}+\left\|\Delta m_{0}\right\|_{L^{2}}^{2}\right]<\alpha,
\end{gathered}
$$

the smooth solution of (3.15) exists on $\mathbb{R}_{+}$and verifies, for every $T>0$

$$
\|\Delta m(T)\|_{L^{2}}^{2}+\frac{\alpha-N(T)}{\lambda} \int_{0}^{T}\|\nabla \Delta m(t)\|_{L^{2}}^{2} \mathrm{~d} t \leqslant\left\|\Delta m_{0}\right\|_{L^{2}}^{2}
$$

where

$$
N(T):=\sup \left\{C^{* *}(\alpha+1)\left[\|\Delta m(t)\|_{L^{2}}+\|\Delta m(t)\|_{L^{2}}^{2}\right] ; t \in[0, T]\right\} .
$$

In particular, we have

$$
\|\Delta m(t)\|_{L^{2}} \leqslant\left\|\Delta m_{0}\right\|_{L^{2}}, \quad \forall t>0 .
$$

Remark 4.1. As a corollary of Theorem 4.2, we have the existence of $2 \mathrm{D}$ global smooth solutions for (3.15) when $\lambda$ is small enough, namely

$$
\lambda\left(1+4\left\|H_{\mathrm{ext}}\right\|_{L^{\infty}\left(\mathbb{R}_{+}, \mathbb{R}^{3}\right)}+2\left\|\dot{H}_{\mathrm{ext}}\right\|_{L^{1}\left(\mathbb{R}_{+}, \mathbb{R}^{3}\right)}\right)<\frac{1}{2 C^{*}(\Omega)},
$$

and for every initial data $m_{0} \in H^{2}\left(\Omega, \mathbb{R}^{3}\right)$ which satisfies $\frac{\partial m_{0}}{\partial \nu}=0$ on $\partial \Omega$ and

$$
\int_{\Omega}\left|\nabla m_{0}\right|^{2}<\frac{1}{2 C^{*}(\Omega)}
$$

This defines an $H^{1}$-neighborhood of uniform magnetizations of fixed size. Similarly, a corollary of Theorem 4.3 is the existence of $3 \mathrm{D}$ global smooth solutions (3.15) when $\lambda$ is small enough, namely

$$
\lambda \leqslant \frac{\alpha}{C^{* *}(\alpha+1)\left(1+\left\|H_{\mathrm{ext}}\right\|_{L^{\infty}\left(\mathbb{R}_{+}\right)}\right)},
$$

and for every $m_{0} \in H^{2}\left(\Omega, \mathbb{R}^{3}\right)$ which satisfies $\frac{\partial m_{0}}{\partial \nu}=0$ on $\partial \Omega$ and

$$
\left\|\Delta m_{0}\right\|_{L^{2}}<\min \left\{1, \frac{\alpha}{2 C^{* *}(\alpha+1)}\right\} .
$$

This defines an $H^{2}$-neighborhood of uniform magnetizations of fixed size.

Remark 4.2. Theorem 4.2 provides the existence of global smooth solutions of (3.15) when $\lambda$ is small enough and for initial conditions in a $H^{1}$-neighborhood of any minimizer $m_{\lambda}^{*}$ of the micromagnetic energy $\mathcal{E}_{\lambda}$, defined by (3.17), when $\lambda>0$ is small enough. Indeed,

$$
\left\|\nabla m_{\lambda}^{*}\right\|_{L^{2}(\Omega)}^{2} \leqslant 2 \lambda \mathcal{E}_{\lambda}\left(m_{\lambda}^{*}\right) \leqslant 2 \lambda \mathcal{E}_{\lambda}\left(e_{1} \chi_{\Omega}\right)=2 \lambda\left\|H_{d}\left(e_{1} \chi_{\Omega}\right)\right\|_{L^{2}(\Omega)}^{2} \leqslant 2 \lambda|\Omega|,
$$

thus (4.1) holds when

$$
\left\|\nabla\left(m_{0}-m_{\lambda}^{*}\right)\right\|_{L^{2}(\Omega)}^{2}+2 \lambda\left[1+2\left\|H_{\mathrm{ext}}\right\|_{L^{\infty}\left(\mathbb{R}_{+}, \mathbb{R}^{3}\right)}+\left\|\dot{H_{\mathrm{ext}}}\right\|_{L^{1}\left(\mathbb{R}_{+}, \mathbb{R}^{3}\right)}\right]<\frac{1}{C^{*}(\Omega)}
$$


Therefore, Theorem 4.2 provides the existence of global smooth solutions of (3.15) when $\lambda$ is small enough, namely

$$
2 \lambda\left[1+2\left\|H_{\mathrm{ext}}\right\|_{L^{\infty}\left(\mathbb{R}_{+}, \mathbb{R}^{3}\right)}+\left\|\dot{H}_{\mathrm{ext}}\right\|_{L^{1}\left(\mathbb{R}_{+}, \mathbb{R}^{3}\right)}\right]<\frac{1}{2 C^{*}(\Omega)},
$$

and for initial conditions in a $H^{1}$-neighborhood of $m_{\lambda}^{*}$, namely

$$
\left\|\nabla\left(m_{0}-m_{\lambda}^{*}\right)\right\|_{L^{2}(\Omega)}^{2} \leqslant \frac{1}{2 C^{*}(\Omega)} .
$$

Theorem 4.2 also provides the existence of global smooth solutions of (3.15) for initial conditions in an $H^{2}$-neighborhood of any minimizer of the micromagnetic energy $\mathcal{E}_{\lambda}$, defined by (3.17), when $\lambda>0$ is small enough. Indeed, on a 3D ellipsoidal domain, for $\lambda$ small enough, the minimizers of the micromagnetic energy $\mathcal{E}_{\lambda}$ are constant in space (see Prop. 5.1 below).

Remark 4.3. In 3D, the existence of global solutions is only proven on ellipsoidal domains on which the stray field has a particular structure. Indeed, on such domains, one has

$$
H_{d}(m)=H_{d}\left(m_{\sharp} \chi_{\Omega}\right)+H_{d}\left(m-m_{\sharp} \chi_{\Omega}\right)
$$

where $H_{d}\left(m_{\sharp} \chi_{\Omega}\right)$ is constant over $\Omega$. In particular, we have the following inequality that will be crucial in the proof

$$
\left\|\nabla H_{d}(m)\right\|_{L^{2}} \leqslant C\|\nabla m\|_{L^{2}},
$$

where $C=C(\Omega)>0$, which is a consequence of Proposition 4.2 below and Poincaré inequality.

These results are more general than [5], Theorems 1.1-1.4:

- in Theorem 4.1, we take into account an external field $H_{\text {ext }}$ which is not considered in [5], Theorems 1.1 and 1.2 ;

- in Theorem 4.2, we take into account the stray field $H_{d}(m)$ and the external field $H_{\text {ext }}$ which are not considered in [5], Theorems 1.3 and 1.4;

- Theorem 4.3 deals with global solutions in a 3D case, this situation is not investigated in [5].

The proofs of Theorems 4.1 and 4.2 follow the ones of [5]. We detail them in order to justify the necessary adaptations due to the presence of $H_{d}$ and $H_{\text {ext }}$. The proof of Theorem 4.3, instead, involves new arguments.

This section is organized as follows. First, in Section 4.1, we recall Sobolev inequalities and some classical properties of the operator $H_{d}$, that will be useful. Then, we prove Theorems 4.1, 4.2 and 4.3 in Sections 4.2, 4.3 and 4.4 respectively.

\subsection{Preliminaries}

Proposition 4.1. Let $\Omega$ be a bounded regular open subset of $\mathbb{R}^{2}$ or $\mathbb{R}^{3}$. There exists $C=C(\Omega)>0$ such that, for every $u \in H^{2}\left(\Omega, S^{2}\right)$ with $\frac{\partial u}{\partial \nu}=0$ on $\partial \Omega$,

$$
\begin{gathered}
\|u\|_{H^{2}(\Omega)} \leqslant C\left(\|u\|_{L^{2}(\Omega)}^{2}+\|\Delta u\|_{L^{2}(\Omega)}^{2}\right)^{1 / 2} \\
\|u\|_{L^{\infty}(\Omega)} \leqslant C\left(\|u\|_{L^{2}(\Omega)}^{2}+\|\Delta u\|_{L^{2}(\Omega)}^{2}\right)^{1 / 2} \\
\|\nabla u\|_{L^{p}} \leqslant C\|\Delta u\|_{L^{2}}, \quad \forall p \in[1,6] \\
\left\|D^{2} u\right\|_{L^{2}} \leqslant C\|\Delta u\|_{L^{2}}
\end{gathered}
$$


and for every $u \in H^{3}\left(\Omega, S^{2}\right)$ with $\frac{\partial \Delta u}{\partial \nu}=\frac{\partial u}{\partial \nu}=0$ on $\partial \Omega$

$$
\begin{gathered}
\|\Delta u\|_{L^{2}} \leqslant C\|\nabla \Delta u\|_{L^{2}}, \\
\left\|D^{2} u\right\|_{L^{3}} \leqslant C\|\Delta u\|_{L^{2}}^{1 / 2}\|\nabla \Delta u\|_{L^{2}}^{1 / 2} .
\end{gathered}
$$

Let $\Omega$ be a bounded regular open subset of $\mathbb{R}^{2}$. There exists $C=C(\Omega)>0$ such that, for every $u \in H^{2}\left(\Omega, S^{2}\right)$ with $\frac{\partial u}{\partial \nu}=0$ on $\partial \Omega$,

$$
\begin{gathered}
\|\nabla u\|_{L^{4}(\Omega)} \leqslant C\|\nabla u\|_{L^{2}(\Omega)}^{1 / 2}\|\Delta u\|_{L^{2}(\Omega)}^{1 / 2}, \\
\|\nabla u\|_{L^{6}(\Omega)} \leqslant C\|\nabla u\|_{L^{2}(\Omega)}^{1 / 3}\|\Delta u\|_{L^{2}(\Omega)}^{2 / 3}
\end{gathered}
$$

and for every $u \in H^{3}\left(\Omega, S^{2}\right)$ with $\frac{\partial \Delta u}{\partial \nu}=\frac{\partial u}{\partial \nu}=0$ on $\partial \Omega$,

$$
\|\nabla u\|_{L^{\infty}(\Omega)} \leqslant C\|\nabla u\|_{L^{2}(\Omega)}^{1 / 2}\|\nabla \Delta u\|_{L^{2}(\Omega)}^{1 / 2} .
$$

Proof of Proposition 4.1. The inequality (4.7) results from the regularity of the operator $A=-\Delta+I$ with domain

$$
D(A):=\left\{u \in H^{2}(\Omega) ; \frac{\partial u}{\partial \nu} \equiv 0 \text { on } \partial \Omega\right\},
$$

and (4.8) follows from (4.7) and the classical embedding $H^{2}(\Omega) \rightarrow L^{\infty}(\Omega)$. The inequality (4.9) with $p=2$ is a consequence of the spectral decomposition

$$
\|\nabla u\|_{L^{2}}^{2}=\sum_{n=0}^{\infty} \lambda_{n}\left|\left\langle u, \varphi_{n}\right\rangle\right|^{2} \leqslant C \sum_{n=0}^{\infty} \lambda_{n}^{2}\left|\left\langle u, \varphi_{n}\right\rangle\right|^{2}=C\|\Delta u\|_{L^{2}}^{2},
$$

where $\left(\varphi_{n}\right)_{n \in \mathbb{N}}$ is defined by (3.3). Thanks to the embedding $H^{1}(\Omega) \rightarrow L^{p}(\Omega)$, with $1 \leq p \leq 6$, the Poincaré inequality and (4.7), we get

$$
\begin{aligned}
\|\nabla u\|_{L^{p}} & =\left\|\nabla\left(u-u_{\sharp}\right)\right\|_{L^{p}} \\
& \leqslant C\left\|u-u_{\sharp}\right\|_{H^{2}} \\
& \leqslant C\left(\|\nabla u\|_{L^{2}}^{2}+\|\Delta u\|_{L^{2}}^{2}\right)^{1 / 2},
\end{aligned}
$$

which leads to (4.9). The inequality (4.10) follows from the regularity of Laplace operator on regular domains, while (4.11) results from a spectral decomposition, in the same way as (4.9). Thanks to the embedding $H^{1 / 2}(\Omega) \rightarrow L^{3}(\Omega)$, we have

$$
\left\|D^{2} u\right\|_{L^{3}} \leqslant C\left(\left(\|u\|_{L^{2}}^{2}+\|\Delta u\|_{L^{2}}^{2}\right)^{1 / 2}+\left(\|u\|_{L^{2}}^{2}+\|\Delta u\|_{L^{2}}^{2}\right)^{1 / 4}\|\nabla \Delta u\|_{L^{2}}^{1 / 2}\right) .
$$

Applying the previous inequality with $u$ replaced by $u-u_{\sharp}$, using Poincaré inequality and (4.9) we get

$$
\begin{aligned}
\left\|D^{2} u\right\|_{L^{3}} & \leqslant C\left(\left(\|\nabla u\|_{L^{2}}^{2}+\|\Delta u\|_{L^{2}}^{2}\right)^{1 / 2}+\left(\|\nabla u\|_{L^{2}}^{2}+\|\Delta u\|_{L^{2}}^{2}\right)^{1 / 4}\|\nabla \Delta u\|_{L^{2}}^{1 / 2}\right) \\
& \leqslant C\left(\|\Delta u\|_{L^{2}}+\|\Delta u\|_{L^{2}}^{1 / 2}\|\nabla \Delta u\|_{L^{2}}^{1 / 2}\right)
\end{aligned}
$$

which gives (4.12) thanks to (4.11). Inequalities (4.13), (4.14), (4.15) are consequences of Galiardo Nirenberg inequalities.

We will also need the following proposition. 
Proposition 4.2 ([5], Lem. 2.3). If $m \in H^{1}(\Omega)$, the restriction of $H_{d}(m)$ to $\Omega$ belongs to $H^{1}(\Omega)$, and there exists $C=C(\Omega)>0$ such that,

$$
\left\|H_{d}(m)\right\|_{H^{1}(\Omega)} \leqslant C\|m\|_{H^{1}(\Omega)} .
$$

\subsection{Local smooth solutions: proof of Theorem 4.1}

This section is dedicated to the proof of Theorem 4.1, following the strategy of [5], Theorem 1.1. This proof is in six steps.

\section{First step: Approximate problem}

We denote by $\mathcal{V}_{n}$ the finite dimensional space built on the $n$ first eigen-functions of $-\Delta$ on $\Omega$ with Neumann conditions, and by $P_{n}$ the orthogonal projection from $L^{2}(\Omega, \mathbb{R})$ on $\mathcal{V}_{n}$. We seek a solution $m_{n} \in C^{1}\left(\left[0, T_{n}\right), \mathcal{V}_{n}\right)$, with $T_{n}>0$ of

$$
\left\{\begin{aligned}
\frac{\partial m_{n}}{\partial t}= & \alpha\left\{\frac{1}{\lambda} \Delta m_{n}+H_{\mathrm{ext}}+\frac{1}{\lambda} P_{n}\left[\left|\nabla m_{n}\right|^{2} m_{n}\right]-P_{n}\left[\left\langle H_{\mathrm{ext}}, m_{n}\right\rangle m_{n}\right]\right. \\
& \left.-P_{n}\left[m_{n} \wedge\left(m_{n} \wedge H_{d}\left(m_{n}\right)\right)\right]\right\}-P_{n}\left[m_{n} \wedge\left(\frac{1}{\lambda} \Delta m_{n}+H_{d}\left(m_{n}\right)+H_{\mathrm{ext}}\right)\right], \\
m_{n}(0)= & P_{n}\left(m_{0}\right) .
\end{aligned}\right.
$$

Thanks to Cauchy-Lipschitz theorem, there exists a unique maximal solution of (4.16) defined on $\left[0, T_{n}\right)$ where $T_{n} \in(0,+\infty]$.

\section{Second step: $L^{2}$ estimate}

Taking the inner product in $L^{2}(\Omega)$ of (4.16) by $m_{n}$, and using (4.7) and (4.8), we get $C=C(\Omega, \alpha, \lambda)>0$ such that

$$
\begin{aligned}
\frac{1}{2} \frac{\mathrm{d}}{\mathrm{d} t}\left[\left\|m_{n}\right\|_{L^{2}}^{2}\right]+\frac{\alpha}{\lambda}\left\|\nabla m_{n}\right\|_{L^{2}}^{2} & =\int_{\Omega} \frac{\alpha}{\lambda}\left|\nabla m_{n}\right|^{2}\left|m_{n}\right|^{2}+\alpha\left\langle H_{\mathrm{ext}}, m_{n}\right\rangle\left(1-\left|m_{n}\right|^{2}\right) \\
& \leqslant C\left(1+\left|H_{\mathrm{ext}}\right|\right)\left[1+\left(\left\|m_{n}\right\|_{L^{2}}^{2}+\left\|\Delta m_{n}\right\|_{L^{2}}^{2}\right)^{2}\right] .
\end{aligned}
$$

Thus, for every $T \in\left(0, T_{n}\right)$, for every $t \in[0, T]$, we have

$$
\left\|m_{n}(t)\right\|_{L^{2}}^{2}+\frac{2 \alpha}{\lambda} \int_{0}^{t}\left\|\nabla m_{n}\right\|_{L^{2}}^{2} \leqslant\left\|m_{0}\right\|_{L^{2}}^{2}+C\left[1+\left\|H_{\mathrm{ext}}\right\|_{L^{\infty}(0, T)}\right] \int_{0}^{t}\left[1+\left(\left\|m_{n}\right\|_{L^{2}}^{2}+\left\|\Delta m_{n}\right\|_{L^{2}}^{2}\right)^{2}\right] .
$$

\section{Third step: $H^{2}$ estimate}

Multiplying (4.16) by $\Delta^{2} m_{n}$ and using integrations by parts, we get

$$
\frac{1}{2} \frac{\mathrm{d}}{\mathrm{d} t}\left[\left\|\Delta m_{n}\right\|_{L^{2}(\Omega)}^{2}\right]+\frac{\alpha}{\lambda}\left\|\nabla \Delta m_{n}\right\|_{L^{2}(\Omega)}^{2}=I_{1}+I_{2}+I_{3}+I_{4}
$$

where

$$
\begin{aligned}
I_{1} & :=-\frac{\alpha}{\lambda} \int_{\Omega}\left\langle\nabla\left(\left|\nabla m_{n}\right|^{2} m_{n}\right), \nabla \Delta m_{n}\right\rangle, \\
I_{2} & :=\frac{1}{\lambda} \int_{\Omega}\left\langle\nabla m_{n} \wedge \Delta m_{n}, \nabla \Delta m_{n}\right\rangle, \\
I_{3} & :=\int_{\Omega} \alpha\left\langle\nabla\left(m_{n} \wedge\left(m_{n} \wedge H_{d}\left(m_{n}\right)\right)\right), \nabla \Delta m_{n}\right\rangle+\left\langle\nabla\left(m_{n} \wedge H_{d}\left(m_{n}\right)\right), \nabla \Delta m_{n}\right\rangle, \\
I_{4} & :=\int_{\Omega} \alpha\left\langle H_{\mathrm{ext}}, \nabla m_{n}\right\rangle\left\langle m_{n}, \nabla \Delta m_{n}\right\rangle+\alpha\left\langle H_{\mathrm{ext}}, m_{n}\right\rangle\left\langle\nabla m_{n}, \nabla \Delta m_{n}\right\rangle+\left\langle\nabla m_{n} \wedge H_{\mathrm{ext}}, \nabla \Delta m_{n}\right\rangle .
\end{aligned}
$$


Working as in [5], third step of the proof of Theorem 1.1, we get $C=C(\Omega, \alpha, \lambda)>0$ such that we can estimate the first three terms as

$$
\left|I_{1}+I_{2}+I_{3}\right| \leqslant C\left[1+\left(\left\|m_{n}\right\|_{L^{2}}^{2}+\left\|\Delta m_{n}\right\|_{L^{2}}^{2}\right)^{5 / 4}\right]\left\|\nabla \Delta m_{n}\right\|_{L^{2}}^{3 / 2} .
$$

For the fourth term, we have, thanks to Cauchy-Schwarz inequality,

$$
\begin{aligned}
\left|I_{4}\right| & =\int_{\Omega}\left(2 \alpha\left|m_{n}\right|+1\right)\left|H_{\text {ext }}\right|\left|\nabla m_{n}\right|\left|\nabla \Delta m_{n}\right| \\
& \leqslant\left(2 \alpha\left\|m_{n}\right\|_{L^{\infty}}+1\right)\left|H_{\text {ext }}\right|\left\|\nabla m_{n}\right\|_{L^{2}}\left\|\nabla \Delta m_{n}\right\|_{L^{2}} .
\end{aligned}
$$

Using (4.7) and (4.8), we get $C=C(\Omega, \alpha)$ such that

$$
\left|I_{4}\right| \leqslant C\left|H_{\mathrm{ext}}\right|\left(1+\left\|m_{n}\right\|_{L^{2}}^{2}+\left\|\Delta m_{n}\right\|_{L^{2}}^{2}\right)\left\|\nabla \Delta m_{n}\right\|_{L^{2}} .
$$

Thus, there exists $C=C(\Omega, \alpha, \lambda)>0$ such that

$$
\begin{aligned}
\frac{1}{2} \frac{\mathrm{d}}{\mathrm{d} t}\left[\left\|\Delta m_{n}\right\|_{L^{2}}^{2}\right]+\frac{\alpha}{\lambda}\left\|\nabla \Delta m_{n}\right\|_{L^{2}}^{2} \leqslant & C\left(1+\left(\left\|m_{n}\right\|_{L^{2}}^{2}+\left\|\Delta m_{n}\right\|_{L^{2}}^{2}\right)^{5 / 4}\right)\left\|\nabla \Delta m_{n}\right\|_{L^{2}}^{3 / 2} \\
& +C\left|H_{\text {ext }}\right|\left(1+\left\|m_{n}\right\|_{L^{2}}^{2}+\left\|\Delta m_{n}\right\|_{L^{2}}^{2}\right)\left\|\nabla \Delta m_{n}\right\|_{L^{2}} \\
\leqslant & \frac{\alpha}{4 \lambda}\|\nabla \Delta m\|_{L^{2}}^{2}+C\left(\frac{4 \lambda}{\alpha}\right)^{3}\left[1+\left(\left\|m_{n}\right\|_{L^{2}}^{2}+\left\|\Delta m_{n}\right\|_{L^{2}}^{2}\right)^{5}\right] \\
& +\frac{\alpha}{4 \lambda}\|\nabla \Delta m\|_{L^{2}}^{2}+C \frac{4 \lambda}{\alpha}\left|H_{\mathrm{ext}}\right|^{2}\left[1+\left(\left\|m_{n}\right\|_{L^{2}}^{2}+\left\|\Delta m_{n}\right\|_{L^{2}}^{2}\right)^{2}\right] .
\end{aligned}
$$

Simplifying the terms containing $\left\|\nabla \Delta m_{n}\right\|_{L^{2}}$, and summing with (4.18), we get, for every $T \in\left(0, T_{n}\right)$, for every $t \in[0, T]$

$$
\begin{aligned}
\frac{\mathrm{d}}{\mathrm{d} t}\left[\left\|m_{n}\right\|_{L^{2}(\Omega)}^{2}+\left\|\Delta m_{n}\right\|_{L^{2}(\Omega)}^{2}\right]+\frac{\alpha}{\lambda}\left[\left\|\nabla m_{n}\right\|_{L^{2}(\Omega)}^{2}+\left\|\nabla \Delta m_{n}\right\|_{L^{2}(\Omega)}^{2}\right] \leqslant \\
C\left(1+\left\|H_{\text {ext }}\right\|_{L^{\infty}(0, T)}^{2}\right)\left(1+\left(\left\|m_{n}\right\|_{L^{2}(\Omega)}^{2}+\left\|\Delta m_{n}\right\|_{L^{2}(\Omega)}^{2}\right)^{5}\right),
\end{aligned}
$$

where $C=C(\Omega, \alpha, \lambda)$. Thus, there exists $T^{*}>0$ such that:

- for every $n$, the solution of the approximate problem (4.16) is defined (at least) on $\left(0, T^{*}\right)$ (see [5], Lem. 2.4);

- for every $T \in\left(0, T^{*}\right),\left(m_{n}\right)_{n \in \mathbb{N}^{*}}$ is bounded in

$$
L^{2}\left((0, T), H^{3}(\Omega)\right) \cap L^{\infty}\left((0, T), H^{2}(\Omega)\right) \cap H^{1}\left((0, T), H^{1}\left(\Omega, \mathbb{R}^{3}\right)\right) .
$$

\section{Fourth step: Convergence}

As in [5], these bounds allow to extract converging subsequences and pass to the limit $n \rightarrow+\infty$. In particular the terms with $H_{\text {ext }}$ do not pose any difficulty. The proof of the conservation of the punctual norm of $m$ is identical to [5].

\section{Fifth step: $L^{2}$-stability}

Let $m_{1}, m_{2}$ be two solutions of (3.15), $T^{*}:=\min \left\{T_{1}^{*}, T_{2}^{*}\right\}, v:=m_{1}-m_{2}$. We prove that, for every $T \in\left(0, T^{*}\right)$, there exists $C>0$ such that

$$
\sup _{t \in[0, T]}\|v(t)\|_{L^{2}(\Omega)} \leqslant C\|v(0)\|_{L^{2}(\Omega)} .
$$


One has

$$
\begin{aligned}
\frac{\partial v}{\partial t}= & \alpha\left[\frac{1}{\lambda} \Delta v+\frac{1}{\lambda}\left|\nabla m_{1}\right|^{2} v+\frac{1}{\lambda}\left(\left|\nabla m_{1}\right|^{2}-\left|\nabla m_{2}\right|^{2}\right) m_{2}\right. \\
& -v \wedge\left(m_{1} \wedge H_{d}\left(m_{1}\right)\right)-m_{2} \wedge\left(v \wedge H_{d}\left(m_{1}\right)\right)-m_{2} \wedge\left(m_{2} \wedge H_{d}(v)\right) \\
& \left.-\left\langle H_{\mathrm{ext}}, v\right\rangle m_{1}-\left\langle H_{\mathrm{ext}}, m_{2}\right\rangle v\right] \\
& +v \wedge\left(\frac{1}{\lambda} \Delta m_{1}+H_{d}\left(m_{1}\right)\right)+m_{2} \wedge\left(\frac{1}{\lambda} \Delta v+H_{d}(v)\right)+v \wedge H_{\mathrm{ext}} .
\end{aligned}
$$

Multiplying this equation by $v$, integrating over $\Omega$, using the fact that $m_{1}, m_{2} \in L^{\infty}\left((0, T), H^{2}\right) \cap L^{2}\left((0, T), H^{3}\right)$, we get $f \in L^{1}(0, T)$ such that, for every $t \in[0, T]$,

$$
\frac{\mathrm{d}}{\mathrm{d} t}\|v\|_{L^{2}}^{2}+\frac{\alpha}{\lambda}\|\nabla v\|_{L^{2}}^{2} \leqslant f(t)\|v\|_{L^{2}}^{2}
$$

We conclude (4.21) thanks to Gronwall Lemma.

\section{Sixth step: $H^{2}$-stability}

With the same notations as in the previous step, we prove that, for every $T \in\left(0, T^{*}\right)$, there exists $C=$ $C(\Omega, \alpha, \lambda)>0$ such that

$$
\sup _{t \in[0, T]}\|v(t)\|_{H^{2}(\Omega)} \leqslant C\|v(0)\|_{H^{2}(\Omega)} .
$$

We go back to the Galerkin approximations. Taking the inner product of the equation on $m_{n}$ with $\Delta^{2} m_{n}$, integrating by parts on $\Omega$, integrating in time between 0 and $t$ and taking the limit when $n$ tends to infinity gives the following inequality (thanks to the lower semi continuity of the norms under the weak topology)

$$
\frac{1}{2}\|\Delta v(t)\|_{L^{2}(\Omega)}^{2}+\frac{\alpha}{\lambda} \int_{0}^{t}\|\nabla \Delta v\|_{L^{2}(\Omega)}^{2} \leqslant \frac{1}{2}\left\|\Delta v_{0}\right\|_{L^{2}(\Omega}^{2}+\int_{0}^{t} \sum_{j=1}^{9} J_{j}(\tau) \mathrm{d} \tau,
$$

where $J_{1}, \ldots, J_{9}$ respectively stand for the following expressions

$$
\begin{aligned}
J_{1} & :=\left|\int_{\Omega} \nabla\left(v \wedge \Delta m_{1}\right) \nabla \Delta v\right|, \\
J_{2} & :=\left|\int_{\Omega} \nabla\left(v \wedge H_{d}\left(m_{1}\right)\right) \nabla \Delta v\right|, \\
J_{3} & :=\left|\int_{\Omega} \nabla\left(m_{2} \wedge \Delta v\right) \nabla \Delta v\right|, \\
J_{4} & :=\left|\int_{\Omega} \nabla\left(m_{2} \wedge H_{d}(v)\right) \nabla \Delta v\right|, \\
J_{5} & :=\left|\int_{\Omega} \nabla\left(\left|\nabla m_{1}\right|^{2} v\right) \nabla \Delta v\right|, \\
J_{6} & :=\left|\int_{\Omega} \nabla\left(\left(\left|\nabla m_{1}\right|^{2}-\left|\nabla m_{2}\right|^{2}\right) m_{2}\right) \nabla \Delta v\right|,
\end{aligned}
$$




$$
\begin{aligned}
J_{7} & :=\left|\int_{\Omega} \nabla\left(v \wedge\left(m_{1} \wedge H_{d}\left(m_{1}\right)\right)\right) \nabla \Delta v\right|, \\
J_{8} & :=\left|\int_{\Omega} \nabla\left(m_{2} \wedge\left(v \wedge H_{d}\left(m_{1}\right)+m_{2} \wedge H_{d}(v)\right)\right) \nabla \Delta v\right|, \\
J_{9} & :=\left|\int_{\Omega} \alpha\left\langle\nabla\left[\left\langle H_{\mathrm{ext}}, v\right\rangle m_{1}\right], \nabla \Delta v\right\rangle-\alpha\left\langle\nabla\left[\left\langle H_{\mathrm{ext}}, m_{2}\right\rangle v\right], \nabla \Delta v\right\rangle-\left\langle\nabla v \wedge H_{\mathrm{ext}}, \nabla \Delta v\right\rangle\right| .
\end{aligned}
$$

Working as in [5], pp. 12-13, there exists $f_{1} \in L^{1}(0, T)$ such that

$$
\left|\sum_{j=1}^{8} J_{j}\right| \leqslant \frac{\alpha}{4 \lambda}\|\nabla \Delta v\|_{L^{2}}^{2}+f_{1}(t)\left(\|v(t)\|_{L^{2}}^{2}+\|\Delta v(t)\|_{L^{2}}^{2}\right) .
$$

For the last term $J_{9}$, we get from Cauchy-Schwarz inequality that

$$
\begin{aligned}
\left|J_{9}\right| \leqslant & \int_{\Omega}\left[\alpha\left(\left|m_{1}\right|+\left|m_{2}\right|\right)+1\right]\left|\nabla v\|\nabla \Delta v\| H_{\text {ext }}\right|+\alpha\left[\left|\nabla m_{1}\right|+\left|\nabla m_{2}\right|\right]\left|v\||| \nabla \Delta v\| H_{\text {ext }}\right| \\
\leqslant & {\left[\alpha\left(\left\|m_{1}\right\|_{L^{\infty}}+\left\|m_{2}\right\|_{L^{\infty}}\right)+1\right]\|\nabla v\|_{L^{2}}\|\nabla \Delta v\|_{L^{2}}\left|H_{\text {ext }}\right| } \\
& +\alpha\left[\left\|\nabla m_{1}\right\|_{L^{\infty}}+\left\|\nabla m_{2}\right\|_{L^{\infty}}\right]\|v\|_{L^{2}}\|\nabla \Delta v\|_{L^{2}}\left|H_{\text {ext }}\right| .
\end{aligned}
$$

Using the embeddings $H^{3}(\Omega) \rightarrow W^{1, \infty}(\Omega), H^{2}(\Omega) \rightarrow H^{1}(\Omega)$ and (4.7), there exists $C=C(\Omega, \alpha)>0$ such that

$$
\left|J_{9}\right| \leqslant C\left[1+\left\|m_{1}\right\|_{H^{3}}+\left\|m_{2}\right\|_{H^{3}}\right]\left|H_{\mathrm{ext}}\right|\left(\|v\|_{L^{2}}^{2}+\|\Delta v\|_{L^{2}}^{2}\right)^{1 / 2}\|\nabla \Delta v\|_{L^{2}}
$$

Since $H_{\text {ext }} \in L^{\infty}(0, T)$ and $m_{1}, m_{2} \in L^{2}\left((0, T), H^{3}\right)$, this proves the existence of $f_{2} \in L^{1}(0, T)$ such that

$$
\left|J_{9}\right| \leqslant \frac{\alpha}{4 \lambda}\|\nabla \Delta v\|_{L^{2}}^{2}+f_{2}(t)\left(\|v\|_{L^{2}}^{2}+\|\nabla \Delta v\|_{L^{2}}^{2}\right) .
$$

Therefore, using (4.23), (4.24), (4.25) we get $f \in L^{1}(0, T)$ such that

$$
\|\Delta v(t)\|_{L^{2}}^{2}+\frac{\alpha}{\lambda} \int_{0}^{t}\|\nabla \Delta v\|_{L^{2}}^{2} \leqslant\left\|\Delta v_{0}\right\|_{L^{2}}^{2}+\int_{0}^{t} f(\tau)\left[\|v(\tau)\|_{L^{2}}^{2}+\|\Delta v(\tau)\|_{L^{2}}^{2}\right] \mathrm{d} \tau .
$$

We conclude (4.22) by applying Gronwall Lemma.

\subsection{D global solutions: proof of Theorem 4.2}

In this section, we give the proof of Theorem 4.2. We follow the strategy of [5], Theorem 1.2, in three steps. Namely, let $m$ be a local solution of (3.15). We prove that, under the assumption (4.1), $m$ is bounded in $L^{\infty}\left((0, T), H^{2}(\Omega)\right)$ for every $T>0$, which gives the conclusion.

\section{First step: Estimate on $\nabla m$}

Multiplying the first equation in (3.15) by $\frac{\partial m}{\partial t}$, we get

$$
\int_{\Omega}\left|\frac{\partial m}{\partial t}\right|^{2}=-\alpha \frac{\mathrm{d}}{\mathrm{d} t}\left[\mathcal{E}_{\lambda}(m)\right]+\int_{\Omega} \alpha\left\langle H_{\mathrm{ext}}, \frac{\partial m}{\partial t}\right\rangle+\left\langle m \wedge H_{\lambda}(m), \frac{\partial m}{\partial t}\right\rangle .
$$


Moreover, the first equation in (3.15) provides

$$
\left|\frac{\partial m}{\partial t}\right|^{2}=\left(1+\alpha^{2}\right)|m \wedge H(m)|^{2}
$$

and thus,

$$
\mathcal{E}_{\lambda}(m(t))+\frac{1}{\alpha}\left(1-\frac{1}{\sqrt{1+\alpha^{2}}}\right) \int_{0}^{t}\left\|\frac{\partial m}{\partial t}\right\|_{L^{2}(\Omega)}^{2} \leqslant \mathcal{E}_{\lambda}\left(m_{0}\right)+\int_{0}^{t}\left\langle H_{\mathrm{ext}}, \frac{\mathrm{d} m_{\sharp}}{\mathrm{d} t}\right\rangle .
$$

Thanks to an integration by parts in the last integral and the property $\left|m_{\sharp}\right| \leqslant 1$, we get

$$
\mathcal{E}_{\lambda}(m(t)) \leqslant \mathcal{E}_{\lambda}\left(m_{0}\right)+2\left\|H_{\text {ext }}\right\|_{L^{\infty}}+\left\|\dot{H}_{\text {ext }}\right\|_{L^{1}}
$$

\section{Second step: Estimate on $\Delta m$}

Multiplying the first equation of (3.15) by $\Delta m$ we get

$$
\frac{1}{2} \frac{\mathrm{d}}{\mathrm{d} t}\|\nabla m\|_{L^{2}}^{2}+\frac{\alpha}{\lambda}\|\Delta m\|_{L^{2}}^{2}=\frac{\alpha}{\lambda}\|\nabla m\|_{L^{4}}^{4}+K_{1}+K_{2},
$$

where

$$
\begin{aligned}
K_{1} & :=\int_{\Omega}-\alpha\left\langle H_{d}(m)-\left\langle H_{d}(m), m\right\rangle m, \Delta m\right\rangle+\left\langle m \wedge H_{d}(m), \Delta m\right\rangle, \\
K_{2} & :=\alpha \int_{\Omega}|\nabla m|^{2}\left\langle H_{\mathrm{ext}}, m\right\rangle .
\end{aligned}
$$

The inequalities (4.13) and (4.26) give

$$
\|\nabla m\|_{L^{4}(\Omega)}^{4} \leqslant C^{*} B_{1}\|\Delta m\|_{L^{2}(\Omega)}^{2}
$$

where $B_{1}$ is the left hand side of (4.1). Thanks to (4.1), there exists $\epsilon>0$ such that $1-\epsilon-C^{*} B_{1}>0$. Using Proposition 4.2 , we can bound $K_{1}$ and $K_{2}$ by

$$
\begin{aligned}
\left|K_{1}\right| & \leqslant(\alpha+1)\left\|H_{d}(m)\right\|_{L^{2}}\|\Delta m\|_{L^{2}} \\
& \leqslant \frac{\epsilon \alpha}{\lambda}\|\Delta m\|_{L^{2}}^{2}+\frac{\lambda}{\epsilon \alpha}(\alpha+1)^{2} \\
\left|K_{2}\right| & \leqslant \alpha\left|H_{\mathrm{ext}}\right|\|\nabla m\|_{L^{2}}^{2} .
\end{aligned}
$$

Thus, we get

$$
\frac{1}{2} \frac{\mathrm{d}}{\mathrm{d} t}\|\nabla m(t)\|_{L^{2}}^{2}+\frac{\alpha}{\lambda}\left(1-\epsilon-C^{*} B_{1}\right)\|\Delta m\|_{L^{2}}^{2} \leqslant \frac{\lambda}{\epsilon \alpha}(\alpha+1)^{2}+\alpha\left|H_{\text {ext }}\right| B_{1}
$$

Integrating in time, we get the existence of a constant $B_{2}=B_{2}\left(\Omega, \alpha, \lambda, \epsilon,\left\|\nabla m_{0}\right\|_{L^{2}},\left\|H_{\text {ext }}\right\|_{L^{\infty}},\left\|\dot{H}_{\text {ext }}\right\|_{L^{1}}\right)>0$ such that

$$
\int_{0}^{t}\|\Delta m\|_{L^{2}}^{2} \leqslant B_{2}\left[\left\|\nabla m_{0}\right\|_{L^{2}}^{2}+t\right]
$$




\section{Third step: Estimate on $\nabla \Delta m_{n}$}

Let us consider the solutions of (4.16). In the $2 \mathrm{D}$ case, it is possible to give better bounds for the right-hand side of (4.19).

Namely, thanks to Cauchy-Schwarz inequality, we have

$$
\begin{aligned}
\left|I_{1}\right| & \leqslant \frac{\alpha}{\lambda} \int_{\Omega} 2\left|D^{2} m_{n}\right|\left|\nabla m_{n} \| m_{n}\right|\left|\nabla \Delta m_{n}\right|+\left|\nabla m_{n}\right|^{3}\left|\nabla \Delta m_{n}\right| \\
& \leqslant \frac{\alpha}{\lambda}\left[2\left\|m_{n}\right\|_{L^{\infty}}\left\|\nabla m_{n}\right\|_{L^{\infty}}\left\|D^{2} m_{n}\right\|_{L^{2}}\left\|\nabla \Delta m_{n}\right\|_{L^{2}}+\left\|\nabla m_{n}\right\|_{L^{6}}^{3}\left\|\nabla \Delta m_{n}\right\|_{L^{2}}\right] .
\end{aligned}
$$

Using (4.10), (4.14) and (4.15), we get $C=C(\Omega)>0$ such that

$$
\left|I_{1}\right| \leqslant \frac{C \alpha}{\lambda}\left[\left\|m_{n}\right\|_{L^{\infty}}\left\|\nabla m_{n}\right\|_{L^{2}}^{1 / 2}\left\|\Delta m_{n}\right\|_{L^{2}}\left\|\nabla \Delta m_{n}\right\|_{L^{2}}^{3 / 2}+\left\|\nabla m_{n}\right\|_{L^{2}}\left\|\Delta m_{n}\right\|_{L^{2}}^{2}\left\|\nabla \Delta m_{n}\right\|_{L^{2}}\right] .
$$

Thus, there exists $C_{1}=C_{1}(\Omega, \alpha, \lambda)>0$ such that

$$
\left|I_{1}\right| \leqslant \frac{\alpha}{8 \lambda}\left\|\nabla \Delta m_{n}\right\|_{L^{2}}^{2}+C_{1}\left[\left\|m_{n}\right\|_{L^{\infty}}^{4}\left\|\nabla m_{n}\right\|_{L^{2}}^{2}\left\|\Delta m_{n}\right\|_{L^{2}}^{4}+\left\|\nabla m_{n}\right\|_{L^{2}}^{2}\left\|\Delta m_{n}\right\|_{L^{2}}^{4}\right] .
$$

For $I_{2}$, we have thanks to Cauchy-Schwarz inequality,

$$
\begin{aligned}
\left|I_{2}\right| & \leqslant \frac{1}{\lambda} \int_{\Omega}\left|\nabla m_{n} \| \Delta m_{n}\right|\left|\nabla \Delta m_{n}\right| \\
& \leqslant \frac{1}{\lambda}\left\|\nabla m_{n}\right\|_{L^{\infty}}\left\|\Delta m_{n}\right\|_{L^{2}}\left\|\nabla \Delta m_{n}\right\|_{L^{2}},
\end{aligned}
$$

and using (4.15), we get $C_{2}=C_{2}(\Omega, \alpha, \lambda)>0$ such that

$$
\begin{aligned}
\left|I_{2}\right| & \leqslant \frac{C}{\lambda}\left\|\nabla m_{n}\right\|_{L^{2}}^{1 / 2}\left\|\Delta m_{n}\right\|_{L^{2}}\left\|\nabla \Delta m_{n}\right\|_{L^{2}}^{3 / 2} \\
& \leqslant \frac{\alpha}{4 \lambda}\left\|\nabla \Delta m_{n}\right\|_{L^{2}}^{2}+C_{2}\left\|\nabla m_{n}\right\|_{L^{2}}^{2}\left\|\Delta m_{n}\right\|_{L^{2}}^{4} .
\end{aligned}
$$

Thanks to Cauchy-Schwarz inequality, we have

$$
\begin{aligned}
\left|I_{3}\right| & \leqslant \int_{\Omega}\left[2 \alpha\left|m_{n}\right|+1\right]\left|\nabla m_{n}\right|\left|H_{d}\left(m_{n}\right)\right|\left|\nabla \Delta m_{n}\right|+\left[\alpha\left|m_{n}\right|+1\right]\left|m_{n} \| \nabla H_{d}\left(m_{n}\right)\right|\left|\nabla \Delta m_{n}\right| \\
& \leqslant\left[2 \alpha\left\|m_{n}\right\|_{L^{\infty}}+1\right]\left[\left\|\nabla m_{n}\right\|_{L^{\infty}}\left\|H_{d}\left(m_{n}\right)\right\|_{L^{2}}\left\|\nabla \Delta m_{n}\right\|_{L^{2}}+\left\|m_{n}\right\|_{L^{\infty}}\left\|\nabla H_{d}\left(m_{n}\right)\right\|_{L^{2}}\left\|\nabla \Delta m_{n}\right\|_{L^{2}}\right]
\end{aligned}
$$

which, using (4.15) and Proposition 4.2, gives a constant $C=C(\Omega)>0$ such that

$$
\left|I_{3}\right| \leqslant C\left[2 \alpha\left\|m_{n}\right\|_{L^{\infty}}+1\right]\left[\left\|\nabla m_{n}\right\|_{L^{2}}^{1 / 2}\left\|m_{n}\right\|_{L^{2}}\left\|\nabla \Delta m_{n}\right\|_{L^{2}}^{3 / 2}+\left\|m_{n}\right\|_{L^{\infty}}\left\|m_{n}\right\|_{H^{1}}\left\|\nabla \Delta m_{n}\right\|_{L^{2}}\right] .
$$

Therefore, there exists $C_{3}=C_{3}(\Omega, \alpha, \lambda)>0$ such that

$$
\left|I_{3}\right| \leqslant \frac{\alpha}{8 \lambda}\left\|\nabla \Delta m_{n}\right\|_{L^{2}}^{2}+C_{3}\left[\left\|m_{n}\right\|_{L^{\infty}}^{4}+1\right]\left[\left\|\nabla m_{n}\right\|_{L^{2}}^{2}\left\|m_{n}\right\|_{L^{2}}^{4}+\left\|m_{n}\right\|_{L^{\infty}}^{2}\left\|m_{n}\right\|_{H^{1}}^{2}\right] .
$$

The last term is estimated thanks to Cauchy-Schwarz inequality. Indeed, one has

$$
\begin{aligned}
\left|I_{4}\right| & \leqslant \int_{\Omega}\left[2 \alpha\left|m_{n}\right|+1\right]\left|H_{\text {ext }}\right|\left|\nabla m_{n} \| \nabla \Delta m_{n}\right| \\
& \leqslant\left[2 \alpha\left\|m_{n}\right\|_{L^{\infty}}+1\right]\left|H_{\text {ext }}\right|\left\|\nabla m_{n}\right\|_{L^{2}}\left\|\nabla \Delta m_{n}\right\|_{L^{2}},
\end{aligned}
$$


and thus, there exists $C_{4}=C_{4}(\Omega, \alpha, \lambda)>0$ such that

$$
\left|I_{4}\right| \leqslant \frac{\alpha}{8 \lambda}\left\|\nabla \Delta m_{n}\right\|_{L^{2}}^{2}+C_{4}\left[\left\|m_{n}\right\|_{L^{\infty}}^{2}+1\right]\left|H_{\mathrm{ext}}\right|^{2}\left\|\nabla m_{n}\right\|_{L^{2}}^{2} .
$$

Putting together (4.19), (4.29), (4.30), (4.31) and (4.32), we get the inequality

$$
\left\|\Delta m_{n}(t)\right\|_{L^{2}}^{2}+\frac{\alpha}{\lambda} \int_{0}^{t}\left\|\nabla \Delta m_{n}\right\|_{L^{2}}^{2} \leqslant\left\|\Delta m_{0}\right\|_{L^{2}}^{2}+\int_{0}^{t} F\left(\left\|m_{n}\right\|_{L^{2}},\left\|m_{n}\right\|_{L^{\infty}},\left\|\nabla m_{n}\right\|_{L^{2}},\left\|\Delta m_{n}\right\|_{L^{2}}\right) .
$$

Considering the liminf when $n \rightarrow+\infty$ in both sides (the norms are lower semi continuous for the weak topology), and using (4.26), we get $C=C\left(\Omega, \alpha, \lambda,\left\|H_{\text {ext }}\right\|_{L^{\infty}},\left\|\dot{H}_{\text {ext }}\right\|_{L^{1}}, m_{0}\right)>0$ such that

$$
\|\Delta m(t)\|_{L^{2}}^{2}+\frac{\alpha}{\lambda} \int_{0}^{t}\|\nabla \Delta m(\tau)\|_{L^{2}}^{2} \mathrm{~d} \tau \leqslant\left\|\Delta m_{0}\right\|_{L^{2}}^{2}+C \int_{0}^{t}\left(1+\|\Delta m(\tau)\|_{L^{2}}^{4}\right) \mathrm{d} \tau .
$$

Finally, under assumption (4.1), inequality (4.28) holds, thus, Gronwall Lemma applied to (4.33) proves that, for every $T>0,\left\|\Delta m_{n}(t)\right\|_{L^{2}}$ is bounded uniformly with respect to $t \in[0, T]$.

\subsection{D Global smooth solutions on ellipsoidal domains: proof of Theorem 4.3}

This section is dedicated to the proof of Theorem 4.3. This proof involves ideas quite different from those in [5].

Let $m$ be a local solution of (3.15). We prove that, under the assumptions (4.2) and (4.3), $m$ belongs to $L^{\infty}\left((0, T), H^{2}\right)$ for every $T>0$, which gives the conclusion.

Let us first notice that it is sufficient to justify (4.4) for every $T>0$. Indeed, let us assume that (4.4) holds for every $T>0$. Under the assumption (4.3), by continuity, we have $N(T)<\alpha$ for $T$ small enough. Let

$$
T^{*}:=\sup \{T>0 ; N(T)<\alpha\} .
$$

We assume $T^{*}<+\infty$. Then, by continuity $N\left(T^{*}\right)=\alpha$, thus (4.4) proves that

$$
\|\Delta m(t)\|_{L^{2}} \leqslant\left\|\Delta m_{0}\right\|_{L^{2}}, \quad \forall t \in\left[0, T^{*}\right] .
$$

Then, thanks to (4.3) and the definition of $N\left(T^{*}\right)$, we have $N\left(T^{*}\right)<\alpha$. This is in contradiction with the definition of $T^{*}$. Therefore, $T^{*}=+\infty$ and $m \in L^{\infty}\left((0,+\infty), H^{2}(\Omega)\right)$.

Now, in order to prove (4.4), we go back to the equality (4.19) for the approximate solutions, and as in the preceding section, we improve the bounds on $I_{1}, I_{2}, I_{3}, I_{4}$ in order to get the conclusion.

Thanks to Holder inequality, we have

$$
\begin{aligned}
\left|I_{1}\right| & \leqslant \frac{\alpha}{\lambda} \int_{\Omega} 2\left|D^{2} m_{n}\right|\left|\nabla m_{n} \| m_{n}\right|\left|\nabla \Delta m_{n}\right|+\left|\nabla m_{n}\right|^{3}\left|\nabla \Delta m_{n}\right| \\
& \leqslant \frac{C \alpha}{\lambda}\left[\left\|D^{2} m_{n}\right\|_{L^{3}}\left\|\nabla m_{n}\right\|_{L^{6}}\left\|m_{n}\right\|_{L^{\infty}}\left\|\nabla \Delta m_{n}\right\|_{L^{2}}+\left\|\nabla m_{n}\right\|_{L^{6}}^{3}\left\|\nabla \Delta m_{n}\right\|_{L^{2}}\right] .
\end{aligned}
$$

Using (4.12) and (4.9), we get a constant $C=C(\Omega)$ such that

$$
\left|I_{1}\right| \leqslant \frac{C \alpha}{\lambda}\left[\left\|m_{n}\right\|_{L^{\infty}}\left\|\Delta m_{n}\right\|_{L^{2}}^{3 / 2}\left\|\nabla \Delta m_{n}\right\|_{L^{2}}^{3 / 2}+\left\|\Delta m_{n}\right\|_{L^{2}}^{3}\left\|\nabla \Delta m_{n}\right\|_{L^{2}}\right],
$$

which, using (4.11), gives the existence of $C_{1}=C_{1}(\Omega)>0$ such that

$$
\left|I_{1}\right| \leqslant \frac{C_{1} \alpha}{\lambda}\left\|\nabla \Delta m_{n}\right\|_{L^{2}}^{2}\left(\left\|m_{n}\right\|_{L^{\infty}}\left\|\Delta m_{n}\right\|_{L^{2}}+\left\|\Delta m_{n}\right\|_{L^{2}}^{2}\right) .
$$


As far as $I_{2}$ is concerned, using Holder inequality, we can estimate

$$
\begin{aligned}
\left|I_{2}\right| & \leqslant \frac{1}{\lambda} \int_{\Omega}\left|\nabla m_{n} \| \Delta m_{n}\right|\left|\nabla \Delta m_{n}\right| \\
& \leqslant \frac{1}{\lambda}\left\|\nabla m_{n}\right\|_{L^{6}}\left\|\Delta m_{n}\right\|_{L^{3}}\left\|\nabla \Delta m_{n}\right\|_{L^{2}} \\
& \leqslant \frac{C}{\lambda}\left\|\Delta m_{n}\right\|_{L^{2}}^{3 / 2}\left\|\nabla \Delta m_{n}\right\|_{L^{2}}^{3 / 2}
\end{aligned}
$$

using (4.9) and (4.12), with a constant $C=C(\Omega)$. Thanks to $(4.11)$, we get $C_{2}=C_{2}(\Omega)$ such that

$$
\left|I_{2}\right| \leqslant \frac{C_{2}}{\lambda}\left\|\Delta m_{n}\right\|_{L^{2}}\left\|\nabla \Delta m_{n}\right\|_{L^{2}}^{2}
$$

For $I_{3}$, Holder inequality leads to

$$
\begin{aligned}
\left|I_{3}\right| & \leqslant \int_{\Omega}\left(2 \alpha\left|m_{n}\right|+1\right)\left(\left|\nabla m_{n}\right|\left|H_{d}\left(m_{n}\right)\right|\left|\nabla \Delta m_{n}\right|+\left|m_{n} \| \nabla H_{d}\left(m_{n}\right)\right|\left|\nabla \Delta m_{n}\right|\right) \\
& \leqslant\left(2 \alpha\left\|m_{n}\right\|_{L^{\infty}}+1\right)\left\|\nabla \Delta m_{n}\right\|_{L^{2}}\left(\left\|\nabla m_{n}\right\|_{L^{6}}\left\|H_{d}\left(m_{n}\right)\right\|_{L^{3}}+\left\|m_{n}\right\|_{L^{\infty}}\left\|\nabla H_{d}\left(m_{n}\right)\right\|_{L^{2}}\right) .
\end{aligned}
$$

Thanks to the embedding $H^{1}(\Omega) \rightarrow L^{3}(\Omega)$ and Proposition 4.2, there exists $C=C(\Omega)>0$ such that

$$
\left\|H_{d}\left(m_{n}\right)\right\|_{L^{3}} \leqslant C\left\|H_{d}\left(m_{n}\right)\right\|_{H^{1}} \leqslant C\left\|m_{n}\right\|_{H^{1}} .
$$

The second part is more subtle. As emphasized in the Remark 4.3, the proof takes advantage of the particular structure of the stray field on an ellipsoid. Indeed, we have

$$
H_{d}(m)=H_{d}\left(m_{\sharp}\right)+H_{d}\left(m-m_{\sharp}\right)
$$

where $H_{d}\left(m_{\sharp}\right)$ is constant over $\Omega$, thus thanks to Proposition 4.2 and Poincaré inequality, there exists $C=$ $C(\Omega)>0$ such that

$$
\left\|\nabla H_{d}(m)\right\|_{L^{2}}=\left\|\nabla H_{d}\left(m-m_{\sharp}\right)\right\|_{L^{2}} \leqslant C\left\|m-m_{\sharp}\right\|_{H^{1}} \leqslant C\|\nabla m\|_{L^{2}} .
$$

Using (4.36), (4.37) and (4.38), we get a constant $C=C(\Omega)>0$ such that

$$
\begin{aligned}
\left|I_{3}\right| & \leqslant C\left(2 \alpha\left\|m_{n}\right\|_{L^{\infty}}+1\right)\left\|\nabla \Delta m_{n}\right\|_{L^{2}}\left(\left\|\Delta m_{n}\right\|_{L^{2}}\left\|m_{n}\right\|_{H^{1}}+\left\|m_{n}\right\|_{L^{\infty}}\left\|\nabla m_{n}\right\|_{L^{2}}\right) \\
& \leqslant C_{3}\left(2 \alpha\left\|m_{n}\right\|_{L^{\infty}}+1\right)\left(\left\|m_{n}\right\|_{H^{1}}+\left\|m_{n}\right\|_{L^{\infty}}\right)\left\|\nabla \Delta m_{n}\right\|_{L^{2}}^{2}
\end{aligned}
$$

using (4.9) and (4.11), and for a constant $C_{3}=C_{3}(\Omega)>0$. Eventually, the last term is treated as follows

$$
\begin{aligned}
\left|I_{4}\right| & \leqslant \int_{\Omega}\left(2 \alpha\left|m_{n}\right|+1\right)\left|H_{\mathrm{ext}}\right|\left|\nabla m_{n}\right|\left|\nabla \Delta m_{n}\right| \\
& \leqslant\left(2 \alpha\left\|m_{n}\right\|_{L^{\infty}}+1\right)\left|H_{\mathrm{ext}}\right|\left\|\nabla m_{n}\right\|_{L^{2}}\left\|\nabla \Delta m_{n}\right\|_{L^{2}} \\
& \leqslant C_{4}\left(2 \alpha\left\|m_{n}\right\|_{L^{\infty}}+1\right)\left|H_{\mathrm{ext}}\right|\left\|\nabla \Delta m_{n}\right\|_{L^{2}}^{2}
\end{aligned}
$$

thanks to (4.9) and (4.11) and where $C_{4}=C_{4}(\Omega)$. 
Finally, integrating (4.19) between $t=0$ and $t=T$, using (4.34), (4.35), (4.39), (4.42), letting $n \rightarrow+\infty$ and using $|m| \equiv 1$, we get $C_{\sharp}=C_{\sharp}(\Omega)>0$ such that

$$
\begin{aligned}
\|\Delta m(T)\|_{L^{2}}^{2}+\frac{2 \alpha}{\lambda} \int_{0}^{T} & \|\nabla \Delta m(t)\|_{L^{2}}^{2} \mathrm{~d} t \leqslant\left\|\Delta m_{0}\right\|_{L^{2}}^{2} \\
& \quad+\int_{0}^{T} C_{\sharp}(\alpha+1)\|\nabla \Delta m\|_{L^{2}}^{2}\left(\frac{1}{\lambda}\left[\|\Delta m\|_{L^{2}}+\|\Delta m\|_{L^{2}}^{2}\right]+\|\nabla m\|_{L^{2}}+1+\left|H_{\text {ext }}\right|\right) .
\end{aligned}
$$

Let us assume that $\lambda \in(0,1)$ is sufficiently small so that

$$
C_{\sharp}(\Omega)(\alpha+1)\left(1+\left\|H_{\text {ext }}\right\|_{L^{\infty}}\right)<\frac{\alpha}{\lambda},
$$

which is a consequence of $(4.3)$ if $C^{* *}$ is chosen so that $C^{* *}>C_{\sharp}$. Then, we have

$$
\begin{aligned}
\|\Delta m(T)\|_{L^{2}}^{2}+\frac{\alpha}{\lambda} \int_{0}^{T} & \|\nabla \Delta m(t)\|_{L^{2}}^{2} \mathrm{~d} t \leqslant\left\|\Delta m_{0}\right\|_{L^{2}}^{2} \\
& +\int_{0}^{T} C_{\sharp}(\alpha+1)\|\nabla \Delta m\|_{L^{2}}^{2}\left(\frac{1}{\lambda}\left[\|\Delta m\|_{L^{2}}+\|\Delta m\|_{L^{2}}^{2}\right]+\|\nabla m\|_{L^{2}}\right) .
\end{aligned}
$$

Since $\lambda \in(0,1)$, thanks to (4.9) with $p=2$, there exists $C=C(\Omega)>0$ such that

$$
\|\nabla m\|_{L^{2}} \leqslant \frac{C}{\lambda}\|\Delta m\|_{L^{2}}
$$

and thus, there exists $C^{* *}=C^{* *}(\Omega)>C_{\sharp}(\Omega)$ such that

$$
\begin{aligned}
\|\Delta m(T)\|_{L^{2}}^{2}+\frac{\alpha}{\lambda} \int_{0}^{T} & \|\nabla \Delta m(t)\|_{L^{2}}^{2} \mathrm{~d} t \leqslant\left\|\Delta m_{0}\right\|_{L^{2}}^{2} \\
& \quad+\int_{0}^{T} C^{* *} \frac{(\alpha+1)}{\lambda}\|\nabla \Delta m\|_{L^{2}}^{2}\left[\|\Delta m\|_{L^{2}}+\|\Delta m\|_{L^{2}}^{2}\right] \mathrm{d} t .
\end{aligned}
$$

This last estimation leads to (4.4).

Remark 4.4. The same result holds with the same proof, when $\Omega$ is a $2 \mathrm{D}$ ellipsoid, instead of a $3 \mathrm{D}$ one. However, the result is weaker that the one of Theorem 4.2 because the initial condition needs to be close to constants in $H^{2}(\Omega)$. In Theorem 4.2 instead, one only needs an initial condition close to constants in $H^{1}(\Omega)$.

\section{EXPONENTIAL STABILIZATION OF UNIFORM MAGNETIZATIONS ON ELLIPSOIDAL DOMAINS}

The goal of this subsection is to propose external magnetic fields $H_{\text {ext }}$ that produce exponential convergence to global minimizers of the energy $\mathcal{E}_{\lambda}$. We consider an ellipsoidal domain $\Omega$ of $\mathbb{R}^{3}$ with $|\Omega|=1, \alpha>0$ and we study (3.15) and (3.16) with $\lambda>0$. On $\Omega$, the stray field generated by a uniform magnetization is constant, thus, up to a change of coordinates, we may assume that

$$
\forall x \in \Omega, \forall \widetilde{m} \in S^{2}, H_{d}\left(\widetilde{m} \chi_{\Omega}\right)(x)=-D \widetilde{m},
$$

where

$$
D=\left(\begin{array}{ccc}
\alpha_{1} & 0 & 0 \\
0 & \alpha_{2} & 0 \\
0 & 0 & \alpha_{3}
\end{array}\right), 0 \leqslant \alpha_{1} \leqslant \alpha_{2} \leqslant \alpha_{3}
$$


Therefore, for non uniform magnetizations the stray field is given by

$$
H_{d}(m)=-D m_{\sharp}+\widetilde{H}_{d}(m),
$$

where

Thus, in view of (1.8), we have

$$
\widetilde{H}_{d}(m):=H_{d}\left(m-m_{\sharp} \chi_{\Omega}\right) .
$$

$$
\left\|\widetilde{H}_{d}(m)\right\|_{L^{2}} \leqslant\left\|m-m_{\sharp}\right\|_{L^{2}}
$$

and Poincaré inequality shows the existence of $C_{d}=C_{d}(\Omega)>0$ such that

$$
\left\|\widetilde{H}_{d}(m)\right\|_{L^{2}} \leqslant C_{d}\|\nabla m\|_{L^{2}}, \quad \forall m \in H^{1}(\Omega) .
$$

We are now in a position to state the results. We begin with the description of the minimizers.

Proposition 5.1. Let $\Omega$ be a $3 D$ ellipsoid. There exists $\lambda^{*}=\lambda^{*}(\Omega)>0$ such that, for every $\lambda \in\left(0, \lambda^{*}\right)$, the micromagnetic energy $\mathcal{E}_{\lambda}$ has exactly two global minimizers: $m \equiv e_{1}$ and $m \equiv-e_{1}$.

Physically speaking, it is clear that taking $H_{\text {ext }}$ parallel to $e_{1}$ should force the magnetization to converge to $e_{1}$. This is indeed the case, and we even show a slightly stronger result. More precisely, we prove that, for $\lambda$ small enough (i.e. for small domains $\Omega_{\lambda}=\sqrt{\lambda} \Omega$ ), the constant external field $H_{\text {ext }}=\beta e_{j}$ forces, locally around $e_{j}$, the exponential convergence of the PDE solutions to $e_{j}$, when the parameter $\beta>0$ is large enough. When $j=1$, we therefore get the exponential stabilization of the global minimizers of the energy.

In that aim, let us introduce the modified energy $E_{\beta, j}$ defined by

$$
E_{\beta, j}(m):=\int_{\Omega} \frac{1}{2 \lambda}|\nabla m|^{2}+\beta\left(1-m_{\sharp}^{(j)}\right)+\frac{1}{2} \int_{\mathbb{R}^{3}}\left|H_{d}(m)\right|^{2}
$$

that measures the $H^{1}$-distance between $m$ and $e_{j}$ since we have

$$
\int_{\Omega}\left|m-e_{j}\right|^{2}=2\left(1-m_{\sharp}^{(j)}\right) .
$$

Proposition 5.2. Let $j \in\{1,2,3\}$. When $H_{\text {ext }}(t) \equiv \beta e_{j}$ for $t \in[0, T]$, the energy $E_{\beta, j}(m)$ is not increasing on $[0, T]$ along the trajectories of (3.15), and we have the estimate on a smooth solution $m$ of (3.15)

$$
\frac{\mathrm{d} E_{\beta, j}}{\mathrm{~d} t} \leqslant-\frac{1}{\alpha}\left(1-\frac{1}{\sqrt{1+\alpha^{2}}}\right) \int_{\Omega}\left|\frac{\partial m}{\partial t}\right|^{2} \text { on }(0, T) .
$$

We now state the main result of this section.

Theorem 5.1. Let $\Omega$ be a $3 D$ ellipsoid and $\alpha>0$. Let $\alpha_{1}, \alpha_{2}, \alpha_{3}, \beta_{1}^{*}, \beta_{2}^{*}, \beta_{3}^{*}$ be the real numbers defined by (5.1), (5.2) and

$$
\beta_{1}^{*}:=\alpha_{1}+\frac{\alpha_{3}-\alpha_{2}}{2 \alpha}, \quad \beta_{2}^{*}:=\alpha_{2}+\frac{\alpha_{3}-\alpha_{1}}{2 \alpha}, \quad \beta_{3}^{*}:=\alpha_{3}+\frac{\alpha_{2}-\alpha_{1}}{2 \alpha} .
$$

Let $j \in\{1,2,3\}$ and $\beta>\beta_{j}^{*}$. There exists $\lambda^{*}=\lambda^{*}(\Omega, \alpha, \beta)>0, \eta=\eta(\Omega, \alpha)>0, \nu=\nu(\Omega, \alpha, \beta, \lambda)>0$, $K(\Omega, \alpha, \beta, \lambda)>0$ such that, for every $m_{0} \in H^{2}\left(\Omega, S^{2}\right)$ with $\frac{\partial m_{0}}{\partial \nu} \equiv 0$ on $\partial \Omega, E_{\beta, j}\left(m_{0}\right) \leqslant \beta$ and

$$
\left\|\Delta m_{0}\right\|_{L^{2}}<\eta
$$

there exists a unique global solution of (3.15) and (3.16) with $H_{\mathrm{ext}} \equiv \beta e_{j}$ which satisfies

$$
\left\|m(t)-e_{j}\right\|_{H^{1}(\Omega)} \leqslant K\left\|m_{0}-e_{j}\right\|_{H^{1}(\Omega)} \mathrm{e}^{-\nu t}
$$


Remark 5.1. The same result holds in $2 \mathrm{D}$ when $\Omega$ is an ellipse and with (5.11) replaced by

$$
\left\|\nabla m_{0}\right\|_{L^{2}}<\eta
$$

Remark 5.2. The constants $\lambda^{*}=\lambda^{*}(\Omega, \alpha, \beta)>0, \eta=\eta(\Omega, \alpha)>0, \nu=\nu(\alpha, \beta, \lambda)>0$, will be explicit in the proof.

Remark 5.3. In view of (4.6), when $\Omega$ is a 3D ellipsoid, $\lambda$ small enough, $\beta$ large enough and $m_{0}$ close enough to a constant in $H^{2}$ then the solution $m(t)$ of (3.15), (3.16) with $H_{\text {ext }}=\beta e_{j}$ converges exponentially to $e_{j}$ in $H^{s}(\Omega)$ for every $s<2$. Indeed, by interpolation, we have for every $\theta \in(0,1]$,

$$
\begin{aligned}
\left\|m(t)-e_{j}\right\|_{H^{\theta+2(1-\theta)}} & \leqslant\left\|m(t)-e_{j}\right\|_{H^{1}}^{\theta}\left\|m(t)-e_{j}\right\|_{H^{2}}^{1-\theta} \\
& \leqslant C(\Omega, \alpha, \beta, \lambda)\left\|m_{0}\right\|_{H^{2}}\left\|m(t)-e_{j}\right\|_{H^{1}}^{\theta} .
\end{aligned}
$$

Remark 5.4. When $\Omega$ is a $3 \mathrm{D}$ ellipsoid, $\lambda$ small enough, $\beta$ large enough, $m_{0} \in H^{4}\left(\Omega, S^{2}\right)$ is in a $H^{2}$-neighborhood of constant magnetizations, and when $H_{\mathrm{ext}} \equiv \beta e_{j}$, then, it can be shown with arguments similar to those used in this article, that smooth solutions belong to

$$
C^{1}\left([0,+\infty), H^{2}\right) \cap C^{0}\left([0,+\infty), H^{4}\right)
$$

and satisfy

$$
\begin{aligned}
& \forall t>0,\left\|\Delta^{2} m(t)\right\|_{L^{2}} \leqslant\left\|\Delta^{2} m_{0}\right\|_{L^{2}}, \\
& \forall t>0, \frac{\mathrm{d}}{\mathrm{d} t}\|\Delta m(t)\|_{L^{2}}^{2}+\frac{\alpha}{\lambda}\|\nabla \Delta m(t)\|_{L^{2}}^{2} \leqslant 0 .
\end{aligned}
$$

Thus, thanks to interpolation theory, the exponential convergence of $m(t)$ to $e_{j}$ holds in $H^{s}(\Omega)$, for every $s<4$. Generalizing the method, one can prove that the convergence in Theorem 5.1 may hold in any $H^{s}(\Omega)$ for $s>0$ provided the initial condition is close enough to constant magnetizations in a space $H^{s^{\prime}}(\Omega)$ where $s^{\prime}>s$ is well chosen.

The rest of this section, devoted to the proof of Theorem 5.1, is organized as follows. In Section 5.1, we prove Propositions 5.1 and 5.2. In Section 5.2, we prove the exponential convergence to zero of $\|\nabla m(t)\|_{L^{2}}$. In Section 5.3, we deduce from the previous result the exponential convergence to zero of $\left|m_{\sharp}(t)-e_{j}\right|$ (or $\left\|m(t)-e_{j}\right\|_{L^{2}}$ in view of (5.8)). Finally, in Section 5.4, we prove Theorem 5.1 and give explicitly the different constants.

\subsection{Proof of Propositions 5.1 and 5.2}

We give here the proofs of Propositions 5.1 and 5.2.

Proof of Proposition 5.1. From (5.3) and (5.4), one has

$$
\left\|H_{d}(m)\right\|_{L^{2}}^{2}=\left\langle D m_{\sharp}, m_{\sharp}\right\rangle+\left\|H_{d}\left(m-m_{\sharp} \chi_{\Omega}\right)\right\|_{L^{2}}^{2}-2 \int_{\Omega}\left\langle H_{d}\left(m_{\sharp} \chi_{\Omega}\right), H_{d}\left(m-m_{\sharp} \chi_{\Omega}\right)\right\rangle .
$$

In the previous equality, the last integral vanishes, since it coincides with

$$
\int_{\Omega}\left\langle H_{d}\left(m_{\sharp} \chi_{\Omega}\right), m-m_{\sharp}\right\rangle
$$


which is zero because $H_{d}\left(m_{\sharp} \chi_{\Omega}\right)$ is constant on $\Omega$. Therefore, we have

$$
\begin{aligned}
\mathcal{E}_{\lambda}(m) & =\int_{\Omega} \frac{1}{2 \lambda}\|\nabla m\|_{L^{2}}^{2}+\frac{1}{2} \int_{\mathbb{R}^{3}}\left\|H_{d}(m)\right\|_{L^{2}}^{2} \\
& \geqslant \frac{1}{2 \lambda}\|\nabla m\|_{L^{2}}^{2}+\frac{\left\langle D m_{\sharp}, m_{\sharp}\right\rangle}{2} .
\end{aligned}
$$

Noticing that $\left|m_{\sharp}\right|^{2}+\frac{1}{|\Omega|}\left\|m-m_{\sharp}\right\|_{L^{2}}^{2}=1$, and using Poincare inequality, we get a constant $C_{P}=C_{P}(\Omega)>0$ such that

$$
\mathcal{E}_{\lambda}(m) \geqslant \frac{1}{2}\left(\frac{C_{P}}{\lambda}-\frac{\alpha_{1}}{|\Omega|}\right)\left\|m-m_{\sharp}\right\|_{L^{2}}^{2}+\frac{1}{2}\left(\left\langle D m_{\sharp}, m_{\sharp}\right\rangle-\alpha_{1}\left|m_{\sharp}\right|^{2}\right)+\frac{\alpha_{1}}{2} .
$$

For $\lambda<\lambda^{*}=\frac{C_{P}|\Omega|}{\alpha_{1}}$ this is always greater than $\mathcal{E}_{\lambda}\left(e_{1} \chi_{\Omega}\right)=\frac{\alpha_{1}}{2}$, with equality if and only if $m=m_{\sharp}=e_{1}$.

Proof of Proposition 5.2. Taking the scalar product in $L^{2}(\Omega)$ of the first equation of $(3.15)$ with $\frac{\partial m}{\partial t}$, we get

$$
\int_{\Omega}\left|\frac{\partial m}{\partial t}\right|^{2}=-\alpha \frac{\mathrm{d} E_{\beta, j}}{\mathrm{~d} t}+\int_{\Omega}\left\langle m \wedge H_{\lambda}(m), \frac{\partial m}{\partial t}\right\rangle .
$$

This gives (5.9) because

$$
\left|\frac{\partial m}{\partial t}\right|^{2}=\left(1+\alpha^{2}\right)|m \wedge H(m)|^{2}
$$

\subsection{Exponential convergence of $\|\nabla m\|_{L^{2}}$}

We now pass to exponential convergence results.

Proposition 5.3. (1) Let $\Omega$ be a $2 D$ ellipsoid with $|\Omega|=1, \alpha>0, H_{\text {ext }} \in L^{\infty}\left(\mathbb{R}_{+}, \mathbb{R}^{3}\right)$ with $\dot{H}_{\text {ext }} \in L^{1}\left(\mathbb{R}_{+}, \mathbb{R}^{3}\right)$, and $c=c(\Omega)>0$ be the largest constant such that

$$
c\|\nabla u\|_{L^{2}(\Omega)} \leqslant\|\Delta u\|_{L^{2}(\Omega)}, \quad \forall u \in H^{2}(\Omega) \text { with } \frac{\partial u}{\partial \nu} \equiv 0 \text { on } \partial \Omega .
$$

There exists $\mathcal{C}^{*}=\mathcal{C}^{*}(\Omega)>0$ such that, for every $\lambda>0$ with

$$
0<\lambda<\frac{\alpha c^{2}}{4\left[\left\|H_{\mathrm{ext}}\right\|_{L^{\infty}}+\mathcal{C}^{*}(\alpha+1)\right]}
$$

for every $m_{0} \in H^{2}\left(\Omega, S^{2}\right)$ with $\frac{\partial m_{0}}{\partial \nu} \equiv 0$ on $\partial \Omega$, and

$$
\left\|\nabla m_{0}\right\|_{L^{2}}^{2}+\lambda\left(\left\|H_{d}\left(m_{0}\right)\right\|_{L^{2}}^{2}+4\left\|H_{\mathrm{ext}}\right\|_{L^{\infty}}+2\left\|\dot{H}_{\mathrm{ext}}\right\|_{L^{1}}\right)<\frac{1}{\mathcal{C}^{*}},
$$

there exists a global solution of (3.15) with initial condition $m(0)=m_{0}$ and this solution satisfies

$$
\|\nabla m(t)\|_{L^{2}} \leqslant\left\|\nabla m_{0}\right\|_{L^{2}} \mathrm{e}^{-\frac{\alpha c^{2}}{2 \lambda} t}, \quad \forall t>0
$$

(2) Let $\Omega$ be a $3 D$ ellipsoidal domain with $|\Omega|=1, \alpha>0, H_{\text {ext }} \in C^{0} \cap L^{\infty}\left(\mathbb{R}_{+}, \mathbb{R}^{3}\right)$ and $c=c(\Omega)>0$ be the largest constant such that (5.13) holds. There exists $\mathcal{C}^{* *}=\mathcal{C}^{* *}(\Omega, \alpha)>0$ such that, for every $\lambda \in(0,1)$ with

$$
\mathcal{C}^{* *}\left(1+\left\|H_{\text {ext }}\right\|_{L^{\infty}}\right)<\frac{\alpha}{\lambda}
$$


for every $m_{0} \in H^{2}\left(\Omega, S^{2}\right)$ with $\frac{\partial m_{0}}{\partial \nu} \equiv 0$ on $\partial \Omega$, and

$$
\mathcal{C}^{* *}\left[\left\|\Delta m_{0}\right\|_{L^{2}}+\left\|\Delta m_{0}\right\|_{L^{2}}^{2}\right]<\alpha,
$$

there exists a global solution of (3.15) with initial condition $m(0)=m_{0}$ and this solution satisfies (5.16).

Remark 5.5. The constants $\mathcal{C}^{*}=\mathcal{C}^{*}(\Omega)$ and $\mathcal{C}^{* *}=\mathcal{C}^{* *}(\Omega, \alpha)$ will be given explicitly.

Remark 5.6. Let us notice that the exponential convergence of $\|\nabla m(t)\|_{L^{2}}$ to zero holds with any $H_{\text {ext }}=$ $H_{\text {ext }}(t)$ in the suitable functional space. One does not need $H_{\text {ext }} \equiv \beta e_{j}$ here.

Proof of Proposition 5.3. First, notice that if $\mathcal{C}^{*}>C^{* 4}$ where $C^{*}$ is as in Theorem $4.2\left(\right.$ resp. $\mathcal{C}^{* *}>C^{* *}(\alpha+1)$, where $C^{* *}$ is as in Thm. 4.3), then, under the assumption (5.15) (resp. (5.17) and (5.18)), then Theorem 4.2 (resp. Thm. 4.3) ensures the existence of global solutions.

The beginning of the proof is the same in the $2 \mathrm{D}$ and $3 \mathrm{D}$ situations. We estimate the behavior of the energy

$$
\widetilde{E}(m):=\frac{1}{2} \int_{\Omega}|\nabla m|^{2}
$$

by taking the scalar product in $L^{2}(\Omega)$ of the first equation of (3.15) with $\Delta m$. We get

$$
\begin{aligned}
\frac{\mathrm{d} \widetilde{E}}{\mathrm{~d} t}= & -\frac{\alpha}{\lambda}\|\Delta m\|_{L^{2}}^{2}+\frac{\alpha}{\lambda}\|\nabla m\|_{L^{4}}^{4}-\int_{\Omega}|\nabla m|^{2}\left\langle H_{\mathrm{ext}}, m\right\rangle \\
& +\int_{\Omega}-\alpha\left\langle\Delta m, H_{d}(m)\right\rangle-\alpha|\nabla m|^{2}\left\langle H_{d}(m), m\right\rangle+\left\langle\Delta m, m \wedge H_{d}(m)\right\rangle .
\end{aligned}
$$

Using $|m| \equiv 1$, we have

$$
\begin{aligned}
-\int_{\Omega}|\nabla m|^{2}\left\langle H_{\text {ext }}, m\right\rangle & \leqslant\left|H_{\text {ext }}\right|\|\nabla m\|_{L^{2}}^{2} \\
& \leqslant \frac{\left|H_{\text {ext }}\right|}{c^{2}}\|\Delta m\|_{L^{2}}^{2} .
\end{aligned}
$$

Next, thanks to an integration by part, the property $\frac{\partial m}{\partial \nu} \equiv 0$ on $\partial \Omega$ leads to

$$
\int_{\Omega} \alpha\left\langle\Delta m, H_{d}(m)\right\rangle+\left\langle\Delta m, m \wedge H_{d}(m)\right\rangle=\int_{\Omega} \alpha\left\langle\Delta m, \widetilde{H}_{d}(m)\right\rangle+\left\langle\Delta m, m \wedge \widetilde{H}_{d}(m)\right\rangle,
$$

and using (5.6), (5.13) and $|m| \equiv 1$, we deduce that

$$
\begin{aligned}
\int_{\Omega} \alpha\left\langle\Delta m, H_{d}(m)\right\rangle+\left\langle\Delta m, m \wedge H_{d}(m)\right\rangle & \leqslant(\alpha+1) C_{d}\|\Delta m\|_{L^{2}}\|\nabla m\|_{L^{2}} \\
& \leqslant \frac{(\alpha+1) C_{d}}{c}\|\Delta m\|_{L^{2}}^{2} .
\end{aligned}
$$

Eventually, Cauchy-Schwarz inequality, (1.8) and (4.9) implies that there exists $C_{1}=C_{1}(\Omega)>0$ such that

$$
\begin{aligned}
\alpha \int_{\Omega}|\nabla m|^{2}\left\langle H_{d}(m), m\right\rangle & \leqslant \alpha \int_{\Omega}|\nabla m|^{2}\left|H_{d}(m)\right| \\
& \leqslant \alpha\|\nabla m\|_{L^{4}}^{2}\left\|H_{d}(m)\right\|_{L^{2}} \\
& \leqslant \alpha C_{1}\|\Delta m\|_{L^{2}}^{2}
\end{aligned}
$$




\section{First case: $\Omega$ is a $2 \mathrm{D}$ ellipse}

In the same way as in the first step of the proof of Theorem 4.2, we get

$$
\mathcal{E}_{\lambda}(m(t)) \leqslant \mathcal{E}_{\lambda}\left(m_{0}\right)+2\left\|H_{\mathrm{ext}}\right\|_{L^{\infty}}+\left\|\dot{H}_{\mathrm{ext}}\right\|_{L^{1}} .
$$

Thanks to (4.13) and (5.23), we have

$$
\begin{aligned}
\frac{\alpha}{\lambda}\|\nabla m\|_{L^{4}}^{4} & \leqslant \frac{\alpha C^{* 4}}{\lambda}\|\nabla m\|_{L^{2}}^{2}\|\Delta m\|_{L^{2}}^{2} \\
& \leqslant \alpha C^{* 4}\left(2 \mathcal{E}_{\lambda}\left(m_{0}\right)+4\left\|H_{\mathrm{ext}}\right\|_{L^{\infty}}+2\left\|\dot{H}_{\mathrm{ext}}\right\|_{L^{1}}\right)\|\Delta m\|_{L^{2}}^{2} .
\end{aligned}
$$

Finally, using (5.19) and (5.20), (5.21), (5.22), (5.24), we get

$$
\frac{\mathrm{d}}{\mathrm{d} t} \widetilde{E}(m) \leqslant-\left[\frac{\alpha}{\lambda}-\alpha C^{* 4}\left(2 \mathcal{E}_{\lambda}\left(m_{0}\right)+4\left\|H_{\mathrm{ext}}\right\|_{L^{\infty}}+2\left\|\dot{H}_{\mathrm{ext}}\right\|_{L^{1}}\right)-\frac{\left|H_{\mathrm{ext}}\right|}{c^{2}}-\frac{(\alpha+1) C_{d}}{c}-\alpha C_{1}\right]\|\Delta m\|_{L^{2}}^{2} .
$$

Let us assume that (5.14) and (5.15) hold with the constant $\mathcal{C}^{*}$ defined by

$$
\mathcal{C}^{*}:=\max \left\{4 C^{* 4} ; C_{d} c+C_{1} c^{2}\right\}
$$

Then, we have

$$
\frac{\mathrm{d} \widetilde{E}}{\mathrm{~d} t} \leqslant-\frac{\alpha c^{2}}{\lambda} \widetilde{E}
$$

which gives the conclusion.

\section{Second case: $\Omega$ is a $3 \mathrm{D}$ ellipsoid}

Thanks to (4.9) and (4.6), we get $C_{2}=C_{2}(\Omega)>0$ such that

$$
\begin{aligned}
\frac{\alpha}{\lambda}\|\nabla m\|_{L^{4}}^{4} & \leqslant \frac{\alpha C_{2}}{\lambda}\|\Delta m\|_{L^{2}}^{4} \\
& \leqslant \frac{\alpha C_{2}}{\lambda}\left\|\Delta m_{0}\right\|_{L^{2}}^{2}\|\Delta m\|_{L^{2}}^{2}
\end{aligned}
$$

Finally, using (5.19) and (5.20), (5.21), (5.22), (5.26), we get

$$
\frac{\mathrm{d}}{\mathrm{d} t} \widetilde{E}(m) \leqslant-\left[\frac{\alpha}{\lambda}-\frac{\alpha}{\lambda} C_{2}\left\|\Delta m_{0}\right\|_{L^{2}}^{2}-\frac{\left|H_{\mathrm{ext}}\right|}{c^{2}}-\frac{(\alpha+1) C_{d}}{c}-\alpha C_{1}\right]\|\Delta m\|_{L^{2}}^{2} .
$$

Let us assume that (5.17) and (5.18) hold with the constant $\mathcal{C}^{* *}$ such that

$$
\mathcal{C}^{* *}:=\max \left\{C^{* *}(\alpha+1), 4 \alpha C_{2}, \frac{4}{c^{2}}, \frac{4(\alpha+1) C_{d}}{c}+4 \alpha C_{1}\right\} .
$$

Then, we have (5.25) which gives the conclusion.

\subsection{Exponential convergence of $m_{\sharp}$}

Now we study the solutions of (3.15) with the external magnetic field $H_{\text {ext }} \equiv \beta e_{j}, j \in\{1,2,3\}$. 
Proposition 5.4. Let $\beta_{k}^{*}, k=1,2,3$ be defined by (5.10). Let $j \in\{1,2,3\}$ and $\beta>\beta_{j}^{*}$. Let $m$ be a global smooth solution of (3.15) with $H_{\mathrm{ext}} \equiv \beta e_{j}$ such that

- $E_{\beta, j}\left(m_{0}\right) \leqslant \beta$;

- there exists $\delta>0$ such that

$$
\|\nabla m(t)\|_{L^{2}} \leqslant\left\|\nabla m_{0}\right\|_{L^{2}} \mathrm{e}^{-\delta t}, \quad \forall t>0 .
$$

Then, there exists $C>0$ (that does not depend on $m$ ) such that

$$
\left|m_{\sharp}(t)-e_{j}\right| \leqslant C\left(\left|m_{\sharp}(0)-e_{j}\right|+\left\|\nabla m_{0}\right\|_{L^{2}}\right) \mathrm{e}^{-\nu t}
$$

where $\nu:=\min \left\{\alpha\left(\beta-\beta_{j}\right), \delta\right\}$.

Proof of Proposition 5.4. We define

$$
\begin{aligned}
f_{j}(t):= & \int_{\Omega}-\frac{\alpha}{\lambda}|\nabla m|^{2} m^{(j)}+\alpha \beta m^{(j)}\left(m^{(j)}-m_{\sharp}^{(j)}\right)+\alpha\left\langle D m_{\sharp}, m_{\sharp}-m\right\rangle m^{(j)} \\
& -\alpha\left[\tilde{H}_{d}(m)^{(j)}-\left\langle\tilde{H}_{d}(m), m\right\rangle m^{(j)}\right]+\left(m \wedge \tilde{H}_{d}(m)\right)^{(j)} .
\end{aligned}
$$

First case: $j=1$

Integrating over $\Omega$ the first component of the first equality of (3.15) with $H_{\text {ext }} \equiv \beta e_{1}$, we get

$$
\frac{\mathrm{d}}{\mathrm{d} t}\left[1-m_{\sharp}^{(1)}\right]=-\alpha \beta\left(1-\left(m_{\sharp}^{(1)}\right)^{2}\right)-\left(\alpha_{3}-\alpha_{2}\right) m_{\sharp}^{(2)} m_{\sharp}^{(3)}+\alpha\left(\alpha_{1}-\left\langle D m_{\sharp}, m_{\sharp}\right\rangle\right) m_{\sharp}^{(1)}+f_{1}(t) .
$$

Since $E_{\beta, 1}\left(m_{0}\right) \leqslant \beta$ and $t \mapsto E_{\beta, 1}[m(t)]$ is not increasing (see Prop. 5.2), we have $m_{\sharp}^{(1)}(t) \geqslant 0, \forall t \geqslant 0$. Thanks to that inequality and $\left|m_{\sharp}(t)\right| \leqslant 1$, we get

$$
\begin{aligned}
\alpha\left(\alpha_{1}-\left\langle D m_{\sharp}, m_{\sharp}\right\rangle\right) m_{\sharp}^{(1)} & \leqslant \alpha \alpha_{1}\left(1-\left|m_{\sharp}^{(1)}\right|^{2}\right) m_{\sharp}^{(1)} \\
& \leqslant \alpha \alpha_{1}\left[1-\left(m_{\sharp}^{(1)}\right)^{2}\right] .
\end{aligned}
$$

Using $\left|m_{\sharp}\right| \leqslant 1$, we get

$$
\begin{aligned}
\left(\alpha_{3}-\alpha_{2}\right)\left|m_{\sharp}^{(2)} m_{\sharp}^{(3)}\right| & \leqslant \frac{\alpha_{3}-\alpha_{2}}{2}\left[\left(m_{\sharp}^{(2)}\right)^{2}+\left(m_{\sharp}^{(3)}\right)^{2}\right] \\
& \leqslant \frac{\alpha_{3}-\alpha_{2}}{2}\left(1-\left(m_{\sharp}^{(1)}\right)^{2}\right) .
\end{aligned}
$$

Thanks to the two previous inequalities, we get

$$
\frac{\mathrm{d}}{\mathrm{d} t}\left[1-m_{\sharp}^{(1)}\right] \leqslant-\alpha \beta_{1}\left[1-m_{\sharp}^{(1)}\right]+f_{1}(t), \text { where } \beta_{1}:=\beta-\beta_{1}^{*} .
$$

Thanks to (5.5) and Poincaré formula, there exists $C_{P}=C_{P}(\Omega)>0$ such that

$$
\begin{aligned}
\alpha\left\langle D m_{\sharp}, m-m_{\sharp}\right\rangle m^{(1)} & \leqslant \alpha \alpha_{3} C_{P}\|\nabla m\|_{L^{2}}, \\
\int_{\Omega}-\alpha\left[\widetilde{H}_{d}(m)-\left\langle\widetilde{H}_{d}(m), m\right\rangle m\right]+\left(m \wedge \widetilde{H}_{d}(m)\right)^{(1)} & \leqslant(\alpha+1) C_{d}\|\nabla m\|_{L^{2}(\Omega)}, \\
\alpha \beta \int_{\Omega} m^{(1)}\left[m^{(1)}-m_{\sharp}^{(1)}\right] & \leqslant \alpha \beta C_{P}\|\nabla m\|_{L^{2}(\Omega)},
\end{aligned}
$$


thus

where

$$
\left|f_{1}(t)\right| \leqslant F_{1} \mathrm{e}^{-\delta t}, \quad \forall t>0
$$

Finally, we get

$$
F_{1}:=\left(\alpha \alpha_{3}+\alpha+1+\alpha \beta\right) C_{P}\left\|\nabla m_{0}\right\|_{L^{2}}+\frac{\alpha}{\lambda}\left\|\nabla m_{0}\right\|_{L^{2}}^{2}
$$

$$
\left(1-m_{\sharp}^{(1)}\right)(t) \leqslant\left(1-m_{\sharp}^{(1)}\right)(0) \mathrm{e}^{-\alpha \beta_{1} t}+\frac{F_{1}}{\left|\alpha \beta_{1}-\delta\right|}\left|\mathrm{e}^{-\alpha \beta_{1} t}-\mathrm{e}^{-\delta t}\right|,
$$

which gives the conclusion.

\section{Second case: $j=2$}

As in the first case, we have

$$
\frac{\mathrm{d}}{\mathrm{d} t}\left[1-m_{\sharp}^{(2)}\right]=-\alpha \beta\left(1-\left(m_{\sharp}^{(2)}\right)^{2}\right)+\left(\alpha_{3}-\alpha_{1}\right) m_{\sharp}^{(1)} m_{\sharp}^{(3)}+\alpha\left(\alpha_{2}-\left\langle D m_{\sharp}, m_{\sharp}\right\rangle\right) m_{\sharp}^{(2)}+f_{2}(t) .
$$

We have

$$
\begin{aligned}
\alpha\left(\alpha_{2}-\left\langle D m_{\sharp}, m_{\sharp}\right\rangle\right) m_{\sharp}^{(2)} & \leqslant \alpha \alpha_{2}\left[1-\left(m_{\sharp}^{(2)}\right)^{2}\right], \\
\left(\alpha_{3}-\alpha_{1}\right)\left|m_{\sharp}^{(2)} m_{\sharp}^{(3)}\right| & \leqslant \frac{\alpha_{3}-\alpha_{1}}{2}\left(1-\left(m_{\sharp}^{(2)}\right)^{2}\right),
\end{aligned}
$$

thus

$$
\frac{\mathrm{d}}{\mathrm{d} t}\left[1-m_{\sharp}^{(2)}\right] \leqslant \alpha \beta_{2}\left[1-m_{\sharp}^{(2)}\right]+f_{2}(t) \text { where } \beta_{2}:=\beta-\beta_{2}^{*} .
$$

We conclude in the same way as in the first case.

Third case: $j=3$

We have

$$
\frac{\mathrm{d}}{\mathrm{d} t}\left[1-m_{\sharp}^{(3)}\right]=-\alpha \beta\left(1-\left(m_{\sharp}^{(3)}\right)^{2}\right)+\left(\alpha_{1}-\alpha_{2}\right) m_{\sharp}^{(1)} m_{\sharp}^{(2)}+\alpha\left(\alpha_{3}-\left\langle D m_{\sharp}, m_{\sharp}\right\rangle\right) m_{\sharp}^{(3)}+f_{3}(t) .
$$

We have

$$
\begin{aligned}
\alpha\left(\alpha_{3}-\left\langle D m_{\sharp}, m_{\sharp}\right\rangle\right) m_{\sharp}^{(3)} & \leqslant \alpha \alpha_{3}\left[1-\left(m_{\sharp}^{(3)}\right)^{2}\right] \\
\left(\alpha_{2}-\alpha_{1}\right)\left|m_{\sharp}^{(1)} m_{\sharp}^{(2)}\right| & \leqslant \frac{\alpha_{2}-\alpha_{1}}{2}\left(1-\left(m_{\sharp}^{(3)}\right)^{2}\right),
\end{aligned}
$$

thus

$$
\frac{\mathrm{d}}{\mathrm{d} t}\left[1-m_{\sharp}^{(3)}\right] \leqslant \alpha \beta_{3}\left[1-m_{\sharp}^{(3)}\right]+f_{3}(t) \text { where } \beta_{3}:=\beta-\beta_{3}^{*} .
$$

We conclude in the same way as in the first case.

\subsection{Conclusion: proof of Theorem 5.1}

In this section, we deduce from Propositions 5.3 and 5.4 the values of the constants in Theorem 5.1.

\section{$2 \mathrm{D}$ case:}

Let $\Omega$ be a $2 \mathrm{D}$ ellipsoid domain, $\alpha>0, \lambda>0, j \in\{1,2,3\}, \beta>\beta_{j}^{*}, m_{0} \in H^{2}\left(\Omega, S^{2}\right)$ with $\partial m_{0} / \partial \nu \equiv 0$ on $\partial \Omega$ and $E_{\beta, j}\left(m_{0}\right) \leqslant \beta$. Let $\mathcal{C}^{*}=\mathcal{C}^{*}(\Omega)$ and $c=c(\Omega)$ be as in Proposition 5.3. We assume

$$
\lambda<\lambda_{*}(\Omega, \alpha, \beta):=\min \left\{\frac{\alpha c^{2}}{4\left[\beta+\mathcal{C}^{*}(\alpha+1)\right]}, \frac{1}{2 \mathcal{C}^{*}[1+4 \beta]}\right\}
$$


and

$$
\left\|\nabla m_{0}\right\|_{L^{2}} \leqslant \eta(\Omega, \alpha):=\frac{1}{\sqrt{2} \mathcal{C}^{*}} .
$$

Then, (5.14) and (5.15) hold, thus (5.16) is satisfied. Applying Proposition 5.4, and using (5.8), we get (5.12) with

$$
\nu=\nu(\Omega, \alpha, \beta, \lambda):=\min \left\{\frac{\alpha c^{2}}{2 \lambda}, \alpha\left(\beta-\beta_{j}^{*}\right)\right\} .
$$

\section{D case:}

Let $\Omega$ be a 3D ellipsoid, $\alpha>0, \lambda \in(0,1), j \in\{1,2,3\}, \beta>\beta_{j}^{*}, m_{0} \in H^{2}\left(\Omega, S^{2}\right)$ with $\partial m_{0} / \partial \nu \equiv 0$ on $\partial \Omega$ and $E_{\beta, j}\left(m_{0}\right) \leqslant \beta$. Let $\mathcal{C}^{* *}=\mathcal{C}^{* *}(\Omega, \alpha)$ and $c=c(\Omega)$ be as in Proposition 5.3. We assume

$$
\lambda<\lambda_{*}(\Omega, \alpha, \beta):=\frac{\alpha}{\mathcal{C}^{*}[1+\beta]}
$$

and

$$
\left\|\Delta m_{0}\right\|_{L^{2}}<\eta(\Omega, \alpha):=\min \left\{1, \frac{\alpha}{2 \mathcal{C}^{* *}}\right\} .
$$

Then, (5.17) and (5.18) hold, thus (5.16) is satisfied. Applying Proposition 5.4, and using (5.8), we get (5.12) with (5.27).

\section{Magnetization sWitching on Ellipsoidal Domains: PDE STUdy}

We use the notation $\beta_{1}^{*}$ defined in (5.10), $H_{\text {ext }}(m)$ defined by $(2.5)$.

Proposition 6.1. Let $\Omega$ be a $2 D$ (resp. 3D) ellipsoid domain, $\alpha>0, \beta>\beta_{1}^{*}, \lambda_{*}=\lambda_{*}(\Omega, \alpha, \beta)$ be as in Theorem 5.1, $T>0, m_{\text {ref }} \in H^{2}\left((0, T), S^{2}\right)$ be such that $-m_{\text {ref }}(0)=m_{\text {ref }}(T)=e_{1}$. We define $H_{\text {ext }} \in L^{\infty}\left(\mathbb{R}_{+}, \mathbb{R}^{3}\right)$ by

$$
H_{\mathrm{ext}}(t):= \begin{cases}H_{\mathrm{ext}}\left(m_{\mathrm{ref}}(t)\right) & \text { if } 0 \leqslant t \leqslant T \\ \beta e_{1} & \text { if } t>T,\end{cases}
$$

where $H_{\text {ext }}\left(m_{\text {ref }}\right)$ is defined by (2.5). There exists $\eta>0$ such that, for every $m_{0} \in H^{2}\left(\Omega, S^{2}\right)$ (resp. $m_{0} \in$ $\left.H^{3}\left(\Omega, S^{2}\right)\right)$ with $\frac{\partial m_{0}}{\partial \nu} \equiv 0$ on $\partial \Omega$ and $\left\|m_{0}+e_{1}\right\|_{H^{1}(\Omega)}<\eta\left(\right.$ resp. $\left.\left\|m_{0}+e_{1}\right\|_{H^{2}(\Omega)}<\eta\right)$, the solution of (3.15) converges exponentially to $e_{1}$ in $H^{1}(\Omega)$.

Proof. We use the continuity with respect to initial conditions for the $C^{0}\left([0, T], H^{2}\right)$-topology and we apply Theorem 5.1 on $(T,+\infty)$.

\section{Conclusion}

In this paper, we have given a first contribution to the mathematical study of the switching of the magnetization inside a three dimensional small particle. This opens improvements in several directions. First, our results are still restricted to ellipsoidal particles and should be generalized to different shapes. It turns out to be not a simple technical difficulty and we plan to investigate this problem in the near future. Also, the case, particularly relevant in practice of an array of particles should be investigated. Indeed, it is absolutely crucial that the particles behave independently, and switching one particle must not perturb the other ones. Eventually, we plan to consider other types of control like the one that models the spin injection technique.

Let us finish by pointing out several differences between Landau-Lifschitz equations and the harmonic maps heat flow into spheres. Although the equations look quite similar, the gyromagnetic term in the magnetic model make them very different from the point of view of mathematical analysis. Indeed, a lot more results are known in the more geometrical case of the harmonic maps equation, like explicit blow-up solutions in finite time [7,9], global regular solutions if the energy is small [17] or if the energy is non-increasing in time [11], or if the initial 
condition takes values in a open half-sphere [13], etc. Non-uniqueness results are known for the heat flow of harmonic maps [8] or for Landau-Lifschitz equations but only when the effective magnetic field consists of the exchange term [1]. Such results are still not known for Landau-Lifschitz equations in full generality and seem very challenging.

Acknowledgements. The authors would like to thank J.-M. Coron for very valuable discussions.

\section{REFERENCES}

[1] F. Alouges and A. Soyeur, On global weak solutions for Landau Lifschitz equations: existence and nonuniqueness. Nonlinear Anal. Theory Meth. Appl. 18 (1992) 1071-1084.

[2] M. Bauer, J. Fassbender, B. Hillebrands and R.L. Stamps, Switching behavior of a Stoner particle beyond the relaxation time limit. Phys. Rev. B 61 (2000) 3410-3416.

[3] G. Bertotti and I. Mayergoyz, The Science of Hysteresis. Academic Press (2006).

[4] W.F. Brown, Micromagnetics. Interscience Publishers (1963).

[5] G. Carbou and P. Fabrie, Regular solutions for Landau-Lifschitz equation in a bounded domain. Diff. Integral Eqns. 14 (2001) 219-229.

[6] G. Carbou, S. Labbé and E. Trélat, Control of travelling walls in a ferromagnetic nanowire. Discrete Contin. Dyn. Syst. Ser. $S 1$ (2008) 51-59.

[7] K.-C. Chang, W.Y. Ding and R. Ye, Finite-time blow-up of the heat flow of harmonic maps from surfaces. J. Differ. Geom. 36 (1992) 507-515.

[8] J.-M. Coron, Nonuniqueness for the heat flow of harmonic maps. Ann. Inst. H. Poincaré Anal. Non Linéaire 7 (1992) 335-344.

[9] J.-M. Coron and J.-M. Ghidaglia, Explosion en temps fini pour le flot des applications harmoniques. C. R. Acad. Sci. Paris Sér. I Math. 308 (1989) 339-344.

[10] A. DeSimone, Hysteresis and imperfection sensitivity in small ferromagnetic particles. Meccanica 30 (1995) 591-603.

[11] A. Freire, Uniqueness for the harmonic map flow in two dimensions. Calc. Var. Partial Differential Equations 3 (1995) 95-105.

[12] A. Hubert and R. Schäfer, Magnetic Domains: The Analysis of Magnetic Microstructures. Springer (1998).

[13] J. Jost, Ein Existenzbeweis für harmonische Abbildungen, die ein Dirichletproblem lösen, mittels der Methode des Wärmeflusses. Manuscripta Math. 34 (1981) 17-25.

[14] R. Kikuchi, On the minimum of magnetization reversal time. J. Appl. Phys. 27 (1956) 1352-1357.

[15] S. Labbé, Simulation numérique du comportement hyperfréquence des matériaux ferromagnétiques. Ph.D. thesis, Université Paris XIII, France (1998).

[16] J.C. Mallinson, Damped gyromagnetic switching. IEEE Trans. Magn. 36 (2000) 1976-1981.

[17] J.-C. Mitteau, Sur les applications harmoniques. J. Differ. Geom. 9 (1974) 41-54.

[18] A. Visintin, On Landau-Lifschitz equations for ferromagnetism. Japan J. Appl. Math. 2 (1985) 69-84. 